\title{
Mesenchymal TNFR2 promotes the development of polyarthritis and comorbid heart valve stenosis
}

\author{
Maria Sakkou, ${ }^{1}$ Panagiotis Chouvardas, ${ }^{1,2}$ Lydia Ntari, ${ }^{1}$ Alejandro Prados, ${ }^{1}$ Kristin Moreth, ${ }^{3}$ \\ Helmut Fuchs, ${ }^{3}$ Valerie Gailus-Durner, ${ }^{3}$ Martin Hrabe de Angelis, ${ }^{3,4,5}$ Maria C. Denis, ${ }^{6}$ \\ Niki Karagianni, ${ }^{6}$ and George Kollias ${ }^{1,2}$ \\ 'Biomedical Sciences Research Center “Alexander Fleming", Vari, Greece. 'Department of Physiology, Medical School, \\ National and Kapodistrian University of Athens, Athens, Greece. ${ }^{3}$ German Mouse Clinic, Institute of Experimental \\ Genetics, Helmholtz Zentrum München, German Research Center for Environmental Health, Neuherberg, Germany. \\ ${ }^{4}$ Chair of Experimental Genetics, School of Life Science Weihenstephan, Technische Universität München, Alte \\ Akademie, Freising, Germany. ${ }^{5}$ German Center for Diabetes Research (DZD), Neuherberg, Germany. ${ }^{6}$ Biomedcode \\ Hellas SA, Vari, Greece.
}

Mesenchymal TNF signaling is etiopathogenic for inflammatory diseases such as rheumatoid arthritis and spondyloarthritis ( $\mathrm{SpA}$ ). The role of Tnfr1 in arthritis has been documented; however, Tnfr2 functions are unknown. Here, we investigate the mesenchymal-specific role of Tnfr2 in the Tnf $f^{1 A R E}$ mouse model of SpA in arthritis and heart valve stenosis comorbidity by cell-specific, Col6a1-cre-driven gene targeting. We find that TNF/Tnfr2 signaling in resident synovial fibroblasts (SFs) and valvular interstitial cells (VICs) is detrimental for both pathologies, pointing to common cellular mechanisms. In contrast, systemic Tnfr2 provides protective signaling, since its complete deletion leads to severe deterioration of both pathologies. SFs and VICs lacking Tnfr2 fail to acquire pathogenic activated phenotypes and display increased expression of antiinflammatory cytokines associated with decreased Akt signaling. Comparative RNA sequencing experiments showed that the majority of the deregulated pathways in Tnf ${ }^{1 A R E}$ mesenchymal-origin SFs and VICs, including proliferation, inflammation, migration, and disease-specific genes, are regulated by Tnfr2; thus, in its absence, they are maintained in a quiescent nonpathogenic state. Our data indicate a pleiotropy of Tnfr2 functions, with mesenchymal Tnfr2 driving cell activation and arthritis/valve stenosis pathogenesis only in the presence of systemic Tnfr2, whereas nonmesenchymal Tnfr2 overcomes this function, providing protective signals and, thus, containing both pathologies.

Conflict of interest: The authors have declared that no conflict of interest exists.

Submitted: November 27, 2017

Accepted: March 2, 2018

Published: April 5, 2018

\section{Reference information:} JCI Insight. 2018;3(7):e98864. https://doi.org/10.1172/jci. insight.98864.

\section{Introduction}

Arthritis and spondyloarthritis ( $\mathrm{SpA})$ are associated primarily with articular inflammation, synovial pannus formation, joint damage, and disability over time. Arthritis is increasingly recognized to comprise a broader syndrome that includes several additional comorbid conditions (1, 2). Cardiovascular (CV) manifestations are the most common comorbid conditions among arthritis patients and have the greatest effect on mortality, with heart failure being a significant contributor to the observed excess mortality (3-5). Our lab has developed various TNF overexpressing mouse models of chronic inflammation, including human ( $\mathrm{Tg} 197$ (6) and mouse (Tnf $\left.{ }^{A R E}\right)$ TNF-transgenic mice $(7,8)$, that display polyarthritis and ankylosing spondylitis disease features. Studies of these models led to the introduction of potentially novel pathogenic principles to explain the cellular basis of TNF function in arthritis, placing synovial fibroblasts (SFs) - mesenchymal-origin cells lining the joint - as sufficient TNF pathogenic targets to initiate and drive the full chronic inflammatory and destructive disease process (9-11)

TNF is a pleiotropic cytokine with important, distinct functions in homeostasis and disease pathogenesis, including inflammation and autoimmune diseases (12). Notably, biologic agents targeting TNF are being successfully used for therapeutic treatment of autoimmune diseases such as rheumatoid arthritis, SpA, and inflammatory bowel disease $(12,13)$. TNF signaling has been also associated with heart valve diseases, myocardial infarction, heart failure, ventricular hypertrophy, interstitial infiltrates, and fibrosis in clinical 
studies performed in patients, yet the cellular responders of TNF signaling in these pathologies are unknown (2-4, 14-18). Moreover, in mouse models of polyarthritis carrying mutations that affect mRNA stability of TNF and result in TNF overexpression, TNF signaling also associates with heart valve disease, though these studies did not provide a mechanism by which cardiac phenotypes develop and did not provide compelling information about the underlying cellular responders of TNF that mediate this pathology (19-21). Valvular diseases involve mechanisms - such as inflammatory cell infiltration accompanied by increased cytokine production, or activation of resident fibroblasts and quiescent valve interstitial cells (VICs) into hyper-proliferative myofibroblasts - that lead to fibrosis in the valve root and leaflets (22-24). Interestingly, mesenchymal cell activation and hyperproliferation are common features of valve disease and arthritis pathophysiology in the TNF-overexpressing mouse models (25-27).

TNF has been implicated in arthritis pathology in mouse models and exerts versatile bioactivities by binding to and activating TNF receptor superfamily member 1a (Tnfr1, Tnfrsfla) and Tnfr2 (Tnfrsf1b) (28). Mesenchymal Tnfr1 and downstream signaling mediators transduce TNF pathogenic functions in arthritis and have been extensively studied in our mouse models; however, nothing is known about the role of mesenchymal Tnfr2 in this process. Tnfr2 signaling on myeloid cells has been associated with homeostatic functions of TNF by affecting cell survival and cell proliferation (29-31). Moreover, we have previously shown that, opposite to Tnfr1, total Tnfr2 ablation in Tnf $f^{A R E}$ mice led to exacerbation of arthritis (7), indicating an overall protective role for Tnfr2 in polyarthritis/SpA disease initiation and progression. Tnfr2 signaling has been shown to regulate Treg function by suppressing Th17 differentiation $(32,33)$, promoting lymphocyte proliferation (34) and monocyte recruitment by endothelial cells $(35,36)$. However, these immune Tnfr2 functions do not influence TnfARE disease progression and cannot explain its beneficial role in arthritis, since TnflARE mice in the immune-deficient Rag1 genetic background lacking mature B and T cells induce full-blown polyarthritis disease features (7). Here, we demonstrate that mesenchymal Tnfr2 is detrimental during arthritis and valve disease pathogenesis upon systemic presence of the receptor, and we analyze the cellular and molecular mechanisms underlying these functions in the articular and valvular mesenchyme.

\section{Results}

Tnf ${ }^{A A R E}$ mice develop TNF-dependent valve stenosis mediated by heart valve mesenchymal cell pathogenic activation. To study the role of Tnfr2 in mesenchymal origin cells in arthritis and heart valve disease, we used mice carrying the Col6a1-cre transgene, which display specificity for mesenchymal cells in the joints and intestine $(9,37)$. In order to characterize the specificity of the Col6a1-cre mouse in the heart valve, we crossed it with the reporter mouse line $R 26^{m T m / m G}(38)$. In this mouse line, all cells express the membrane-targeted Tomato sequence; however, upon cre-mediated recombination, this sequence is excised, enabling the expression of membrane eGFP. Samples from the ankle joint and the aortic heart valve of these mice showed expression of GFP in the mesenchymal compartment (Figure 1A). Specifically, in the ankle joint, Col6a1-cre is strongly expressed in the synovial membrane, bone cartilage, and skeletal muscle, whereas in the heart valve, expression is limited in the aortic valve leaflets that have also been reported to contain mainly mesenchymal-origin cells, the VICs $(23,39)$. To further investigate the identity of these cells, we first examined whether they colocalize with Col6a1-expressing cells, a protein that is expressed by mesenchymal cells in connective tissues and is crucial for structural integrity and cellular adhesion. All of the $\mathrm{GFP}^{+}$cells in the synovial membrane of the ankle joint (Supplemental Figure 1, A and B; supplemental material available online with this article; https://doi.org/10.1172/ jci.insight.98864DS1) and the aortic valve leaflets (Supplemental Figure 1, C and D) expressed Col6a1. $\mathrm{GFP}^{+}$cells in the articular cartilage (Supplemental Figure 1, A and B) are chondrocytes and, thus, devoid of Col6a1 as well as many other SF-specific markers expression (M. Armaka, Biomedical Sciences Research Center; unpublished observations). Furthermore, FACS analysis on freshly isolated (Supplemental Figure 1, E and G) and 10-day-cultured (Supplemental Figure 1, F and H) SFs and VICs from Col6a1-cre-R2 $6^{m T / m G}$ mice verified that they do not express markers of hematopoietic (Cd45) and endothelial origin (Cd31). However, they express a combination of known surface and intracellular mesenchymal cell markers, such as CD29, CD90, Gp38, ICAM, Vcam-1, and vimentin (Supplemental Figure 1, E-H). From this analysis, it is evident that the Col6a1-cre mouse targets mainly Col6a ${ }^{+}$fibroblast-like cells in the ankle joint and the valvular leaflets that express all the known SF and VIC markers under homeostatic conditions. 
H\&E staining of the aortic heart valves revealed that, indeed, Tnf $f^{\mid A R E /+}$ mice progressively develop stenosis of the aortic valve leaflets, starting at 8 weeks of age (data not shown) with $100 \%$ incidence (Figure 1B). By the age of 16 weeks, the aortic root and valve leaflets were grossly thickened to approximately 10-15 times compared with WT littermates in all the Tnf $f^{A R E /+}$ mice examined (Figure 1B). Interestingly, the thickened valves displayed very little immune cell infiltration, as observed by immunocytochemistry (Supplemental Figure 2, A-D), but they developed marked fibrosis (Figure 1C). To investigate the implications of the thickened/fibrotic aortic valve of Tnf ${ }^{A A R E /+}$ mice in their cardiac function, we performed echocardiographic analysis and ECGs. Heart rate (HR) of TnflARE/+ mice and cardiac output at 4 months of age were significantly reduced compared with WT littermates, indicating cardiac dysfunction (Figure 1, D and E). Furthermore, Doppler examination showed that Tnf ${ }^{\mid A R E /+}$ mice display a mild but significant reduction in their calculated left ventricular ejection fraction (LVEF) (Figure 1F) and higher peak aortic valve flow (AoV) (Figure 1G) compared with control animals, indicating aortic valve stenosis (40-42). Interestingly, Tnf ${ }^{A A R E /+}$ mice also displayed prolonged HR corrected time from the start of the $\mathrm{Q}$ wave to the end of the T wave on the ECG trace (QTc) interval prolongation (Figure $1 \mathrm{H})$, which is a predictor of fatal ventricular arrhythmias (43). In summary, Tnf ${ }^{A R E /+}$ mice showed signs of impaired cardiac function compared with WT. Taken together these data, point to a detrimental effect in cardiac function of the observed thickened valve leaflets of Tnf 1 ARE/+ mice, resembling the clinical signs of aortic valve stenosis pathology (44), a common comorbid condition of Ankylosing spondylitis patients $(17,18)$.

Interestingly, an anti-TNF, etanercept (enbrel), and prophylactic regimen in $T \mathrm{f}^{A A R E /+}$ mice upon disease initiation - lasting for 10 weeks - prevented the development of aortic valve stenosis and fibrosis (Supplemental Figure 3, A and B), indicating that, similar to arthritis pathogenesis, TNF is causal for the heart valve stenosis. Furthermore, to investigate whether Col6a $1^{+}$mesenchymal cells of the heart play a primary role in Tnf $f^{A R E}$ heart valve etiopathogenesis, Tnfr1 was ablated by using the Tnfrsfla ${ }^{f / f}$ line combined with the Col6a1-cre line (Col6a1-cre; Tnfrsf1afff or Tnfrsf1a ${ }^{M C K O}$ ). Histological examination of Tnf ${ }^{\text {ARE/+ }}$; Tnfrsf1a ${ }^{\text {MCKO }}$ aortic valves at the age of 16 weeks revealed no signs of the disease (Supplemental Figure 3C). The valve leaflets and root exhibited comparable thickness with physiological conditions, and no excessive fibrosis was detected (Supplemental Figure 3, D and E). Overall, these findings indicate that TNF is causal to arthritis and heart valve stenosis comorbidity in TnfARE/+ mice and that Col6a1-cre ${ }^{+}$mesenchymal cells act as primary TNF responders in mediating disease initiation and progression.

Next, we crossed the Tnf ${ }^{\mid A R E /+}$ polyarthritis/SpA mouse model to the mesenchymal reporter Col6a1cre; $R 26^{m T m / m G}$ mouse line in order to investigate possible associations between mesenchymal Col6a1$\mathrm{cre}^{+}$cell functions downstream of TNF and aortic stenosis. We observed a dramatic increase of $\mathrm{GFP}^{+}$ cells comprising SFs of the synovial membrane in the joint and VICs in the valve leaflets (Figure 1I), pointing to a central role of SFs and VICs in tissue hyperplasia and disease mechanisms. The increased number of $\mathrm{GFP}^{+}$cells points to a common causal, mesenchymal pathogenic axis underlying etiology of arthritis and valve stenosis.

Ablation of mesenchymal Tnfr2 in Tnfrsf $1 b^{M C K O}$ mice results in suppression of arthritis and heart valve stenosis comorbidity in the TnflARE/+ arthritis/SpA model. To assess the mesenchymal-specific function of Tnfr2 in arthritis and valve stenosis, mice carrying the loxP-flanked Tnfrsflb allele, originally reported here, were crossed with Col6a1-cre mice to achieve Tnfr2 ablation specifically in SFs and VICs (Supplemental Figure 4). In addition, mice carrying a complete Tnfr2 deletion (i.e., Tnfr2-deficient mice) were produced by crossing Tnfrsflfff mice with the Deleter-cre mouse line (Tnfrsf $1 b^{D / D}$ ) (45). Tnfr2-deficient, Tnfrsf $1 b^{D / D}$, and mesenchymal-specific deficient Col6a1-cre-Tnfrsf1bff $\left(\right.$ Tnfrsf $\left.1 b^{M C K O}\right)$ mice were born at the expected Mendelian ratio, were viable, were fertile, and didn't display any spontaneous phenotypes (data not shown). Efficiency and specificity of Tnfr2 deletion in SFs and VICs was verified by IHC for Tnfr2 in Colba1-cre; R26 $6^{m T m / m G}$ mesenchymal reporter mice, Col6a1-cre; $R 26^{m T m / m G}-T_{n f r s f 1 b^{f / f}}\left(R 26^{m T m / m G} ; T_{n f r s f 1 b^{M C K O}}\right)$ mice with SFs/VICs-specific Tnfr2 ablation, and Tnfrsf $1 b^{D / D}$ Tnfr2-deficient mice in heart valves (Supplemental Figure 5, A-C) and ankle joint synovium membrane (Supplemental Figure 5, D-F). Tnfr2 is highly expressed in both SFs and VICs, as depicted by the colocalization with $\mathrm{GFP}^{+}$cells, and exerts high deletion efficiency in both sites. Western blot analysis of isolated cultured cells for Tnfr 2 expression showed that the receptor is $100 \%$ deleted in Tnfrsf $1 b^{D / D}$ cells, whereas Tnfrsf $1 b^{M C K O}$ VICs and SFs display a $70.2 \%$ and $76.5 \%$ protein depletion, respectively, due mainly to Col6a1-cre efficiency (46) and to the cell culture purity (Supplemental Figure 5, G-I). 

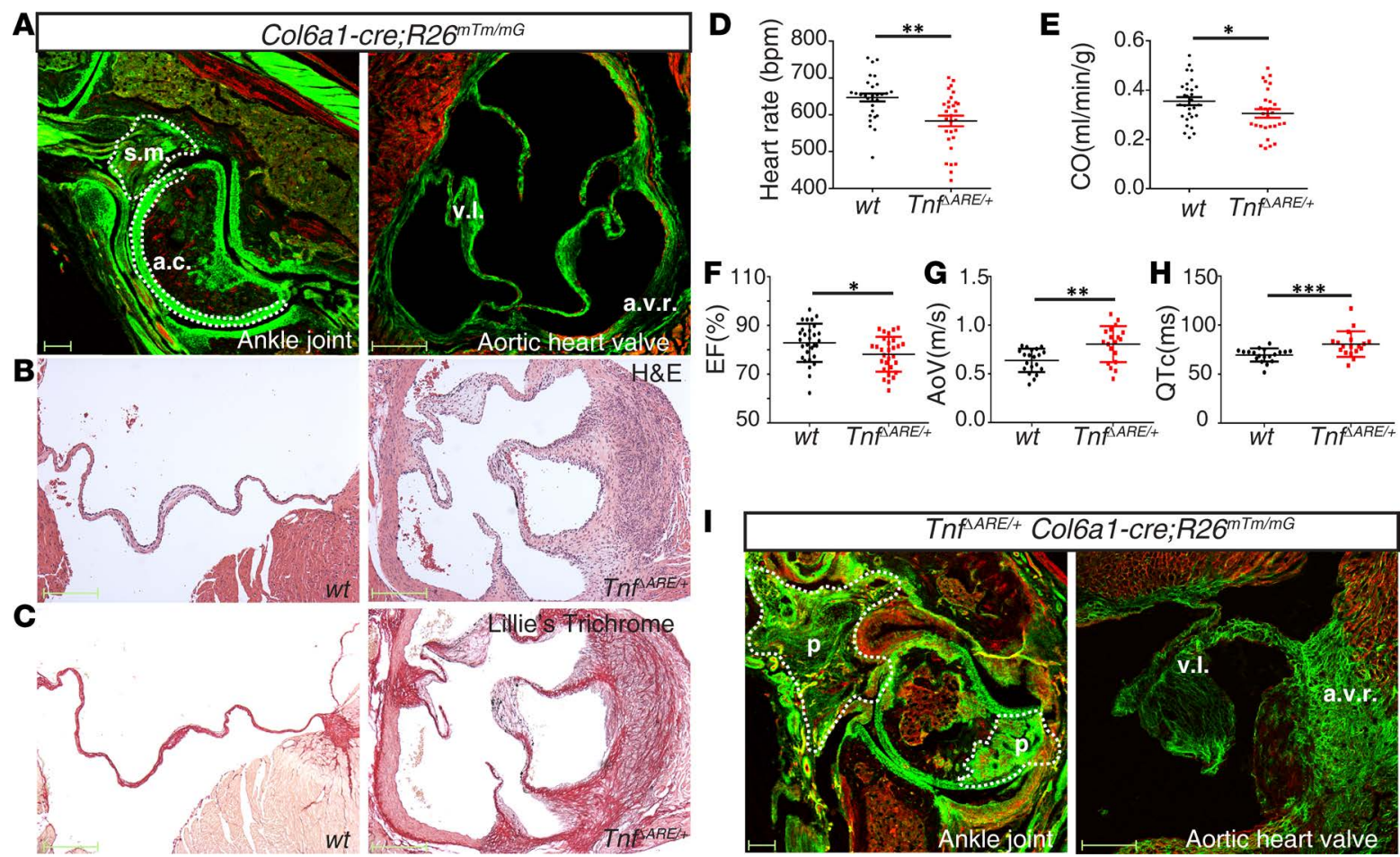

Figure 1. Collagen 6a1-cre+ (Col6a1-cre+) cells are present in the ankle synovium and the aortic valve leaflets in the heart, where they colocalize with cardiac valvular interstitial cells (VICs). Representative images of ankle joint and aortic valve leaflets from Col6a1-cre;R26 ${ }^{\mathrm{mTm} / \mathrm{mG}} \mathrm{mice}(\mathbf{A})$ indicating cre-mediated GFP expression. Representative heart transverse sections stained with H\&E and Lillie's trichrome staining from WT and $T n f^{\triangle A R E /+}$ mice (B and $\left.\mathbf{C}\right)$. Heart rate (D), cardiac output (E), ejection fraction (F), aortic peak velocity (G), and QTc interval quantifications $(\mathbf{H})$, measured by ECG and Doppler echocardiography in 4 months. WT and Tnf ${ }^{\triangle A R E /+}$ mice as indicated $\left(n=29\right.$ mice per genotype), Student's $t$ test, ${ }^{*} P<0.05$, ${ }^{* *} P<$ $0.01,{ }^{* *} P<0.001$. (I) Representative images of ankle joint and aortic heart valve leaflets from ${ }^{*} \mathrm{ff}^{A A R E /+} ;$ Col6a1-cre; $R 26^{\mathrm{mTm} / \mathrm{mC}}$ mice showing increased number of GFP+ cells in both the inflamed ankle joint and the aortic valve (D). Scale bars: $200 \mu \mathrm{m}$. s.m., synovial membrane; a.c., articular cartilage, p, pannus; v.I., valve leaflets; a.v.r., aortic valve root; CO, cardiac output; EF, ejection fraction; AoV, aortic peak velocity; QTc, time from the start of the Q wave to the end of the T wave on the ECG.

TnfARE ${ }^{\text {A+ }}$ Thfrsf $1 b^{M C K O}$ mice displayed significantly reduced clinical features of arthritis. Histopathological evaluation of mice at 16 weeks of age showed a marked amelioration of the disease index characterized by significant reduction of pannus formation and cartilage/bone destruction (Figure 2, A-C). In contrast, Tnf IARE/+ mice bred in total Tnfr2-deficiency background displayed a more aggressive and destructive type of arthritis, exacerbated synovial hyperplasia, and bone destruction, as previously

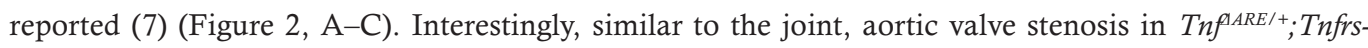
$\mathrm{flb}^{\text {МСКO }}$ mice displayed a strong amelioration including a significant decrease of the leaflets surface area and a marked reduction of fibrosis (Figure 2, D-F). However, notably, Tnfr2-deficient TnfARE/+; Tnfrs$f 1 b^{D / D}$ mice showed an exacerbated pathology characterized by significant increase of the valve leaflet surface area and fibrosis (Figure 2, D-F), thus correlating with arthritis. In both joint and heart valve leaflets of $T n f^{\mid A R E /+} ; \operatorname{Tnfrs} f 1 b^{D / D}$ mice, disease-manifestation took place at earlier time points as compared with TnflARE/+ mice (data not shown). Moreover, interestingly, arthritis and valve stenosis pathologies in Tnf $^{A A R E /+}$; Tnfrsf $1 b^{D / D}$ mice seems to be changed compared with Tnf ${ }^{A R E /+}$ mice since a marked increase of

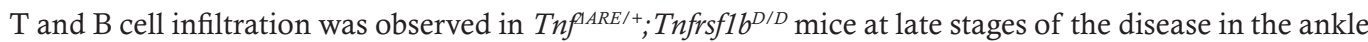
joint (Supplemental Figure 6, A-C) and the aortic valve leaflets (Supplemental Figure 6, D-F) compared with Tnf ${ }^{A A R E /+}$ mice. These data strongly suggest that disease exacerbation in $T n f^{\mid A R E /+} ;$ Tnfrsf $1 b^{D / D}$ mice is caused by the highly proinflammatory milieu in both tissues, developing in the absence of the protective Tnfr2 functions on nonmesenchymal cell types. However, apparently, in the presence of protective Tnfr2 in nonmesenchymal cells, a pathogenic function of the same receptor is expressed in the mesenchymal compartment of both the joint and aortic valve leaflets. Taken together, these results point to a differential role of Tnfr2 in modulating mesenchymal vs. nonmesenchymal cell activation, with important consequences in regulating pathophysiology of arthritic and heart valve disease. 


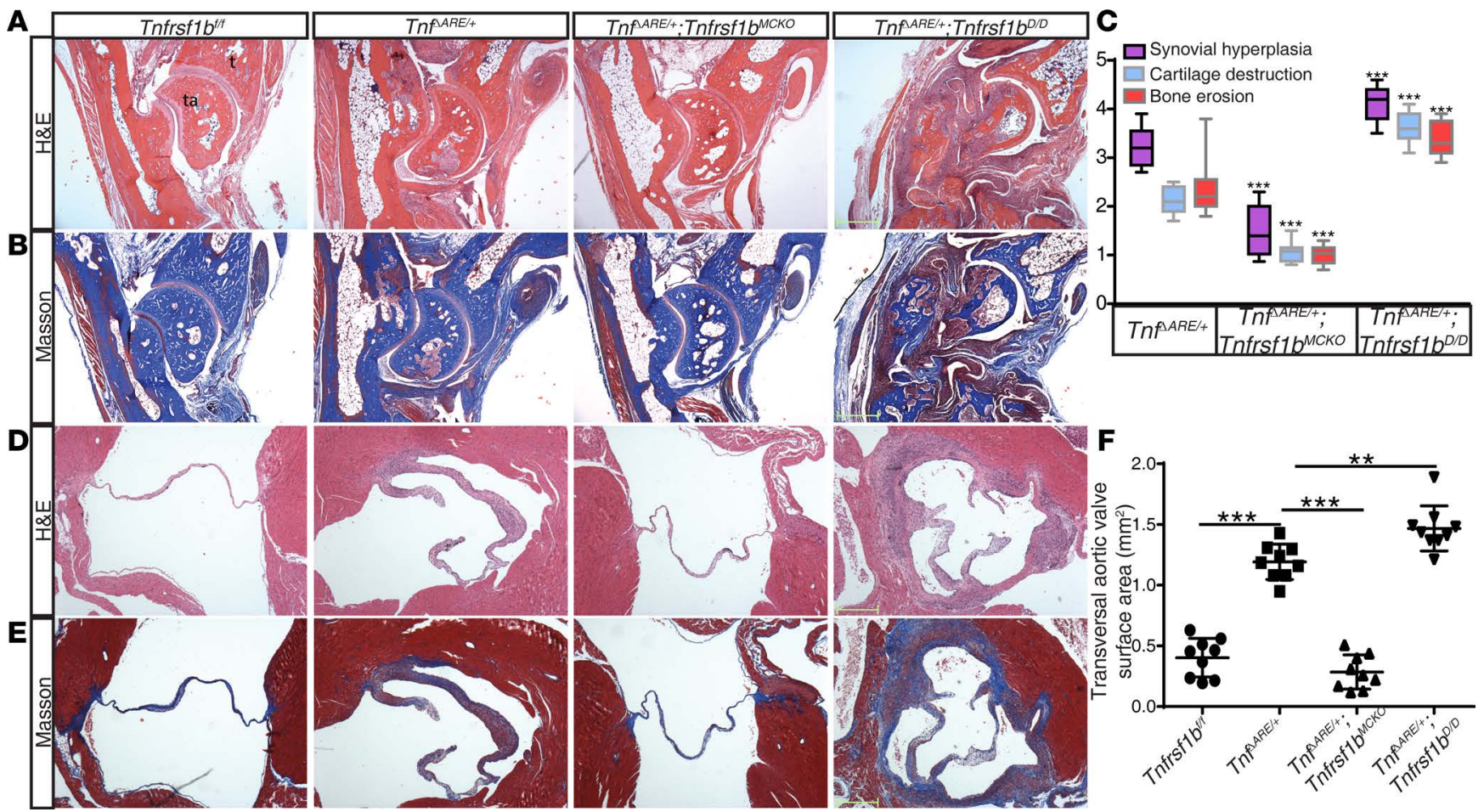

Figure 2. Ablation of mesenchymal Tnfr2 leads to suppression of arthritis and heart valve disease, whereas total ablation of Tnfr2 exacerbates both disease manifestations. Representative serial histological sections at the central axis of ankle joints containing the talus (ta) and tibia ( $t$ ) bones stained with H\&E (A) and Masson's trichrome staining (B). Histopathological scoring for arthritis as measured with a combination of histological stainings; the data are presented as means \pm SEM ( $n=9$ for each group); and 2-way ANOVA with Tukey's multiple comparison tests were used to analyze groups for statistical significance (C). Representative heart transverse sections performed at the level of the aortic valve, stained with H\&E (D) and Masson's trichrome staining (E). Scale bars: $500 \mu \mathrm{m}(\mathbf{A}$ and $\mathbf{B})$ and $100 \mu \mathrm{m}$ ( $\mathbf{D}$ and $\mathbf{E})$. Heart valve stenosis quantification performed by measuring the collagen area stained by Masson using ImageJ (F). Quantifications presented have been performed on 16-week-old mice, the data are presented as means \pm SEM ( $n=9$ for each group), 1-way ANOVA with Bonferroni's multiple comparison tests were used to analyze groups for statistical significance $\left(^{* *} P<0.001,{ }^{* * *} P<0.0001\right)$.

Mesenchymal Tnfr2 directly regulates pathogenic SF and VIC activation. Next, we tested whether Tnfr2 influences mesenchymal pathogenic cell activation in the joint and the heart valve by measuring, in vivo and ex vivo, the expression of well-characterized, disease-specific activation cell markers in Tnf ${ }^{A R E /+}$; Tnfrsf $1 b$ ${ }^{M C K O}$ and $T_{n} f^{A R E /+} ;$ Tnfrsf $1 b^{D / D}$ mice. Adhesion molecules, including ICAM and Vcam-1, are constitutively expressed by SFs at low levels; however, in arthritis, their expression is potently enhanced by TNF and Il-1. Immunostaining and FACS analysis of crude joint cell preparations indicated that double $\mathrm{Cd} 45^{-} / \mathrm{Cd} 31^{-}$ $\mathrm{ICAM}^{+}$cells (Figure 3, A and B, and Supplemental Figure 7, A and B), which are highly upregulated in

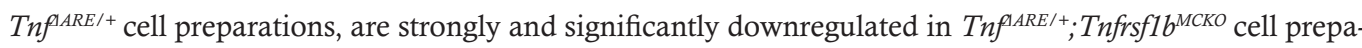
rations, closer to WT control levels (Figure 3, A and B). In contrast, Tnf ${ }^{A R E /+}$; Tnfrsf $1 b^{D / D}$ percentages of gated $\mathrm{Cd} 45^{-} / \mathrm{Cd} 31^{-} \mathrm{ICAM}^{+}$cells were significantly upregulated compared with Tnf ${ }^{A R E /+}$ ones. Quantification

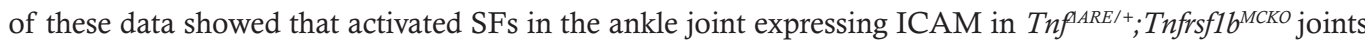
were on average $20 \%$ less abundant, whereas $\operatorname{Tnf}^{A A R E /+} ; \operatorname{Tnfrs} f 1 b^{D / D}$ were $10 \%$ more abundant compared with $\operatorname{Tnf}^{\text {ARE } /+}$ joints (Figure $3 \mathrm{~B}$ ). These results point to a positive correlation between number of pathogenically activated SFs expressing ICAM in the joint and arthritis severity (Figure 2E).

Interestingly, heart valve fibroblasts in pathogenic situations have been shown, similar to SFs, to become activated and adopt a myofibroblast phenotype $(47,48)$, to hyper-proliferate, to acquire a migratory smooth muscle cell-like behavior, and to increase secretion of ECM components, mainly collagen (49, 50). Based on the observation that Tnf ${ }^{A R E /+}$ mice display aortic valve fibrosis, we examined whether, similar to the SFs, heart valve fibroblasts became activated during disease manifestation. Therefore, aortic valve crude cell preparations gated for $\mathrm{Cd} 45^{-} / \mathrm{Cd} 31^{-}$(Supplemental Figure 7A) were stained for vimentin, a well-established VIC marker, and $\alpha$-SMA, a myofibroblast activation marker. Interestingly, similar to the joint, $\operatorname{Tn}^{\rho A R E /+} ; T_{-}$

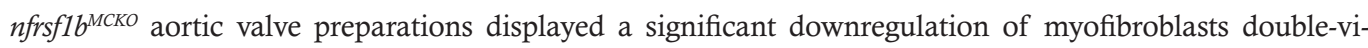
mentin $^{+} / \alpha-\mathrm{SMA}^{+}$cells, whereas $\operatorname{Tnf}^{\mid A R E /+} ; \operatorname{Tnfrs} f 1 b^{D / D}$ aortic valve preparations contained similar percentages 
A

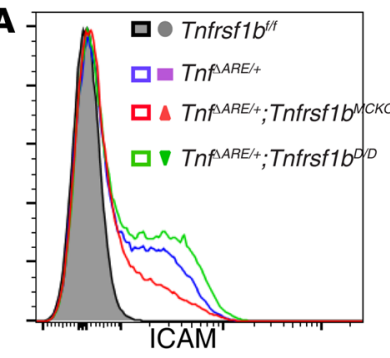

B

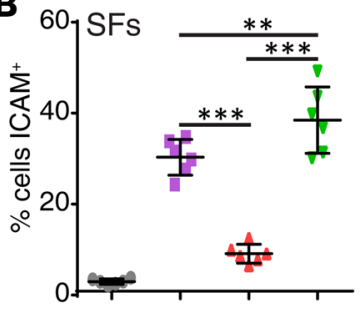

C.

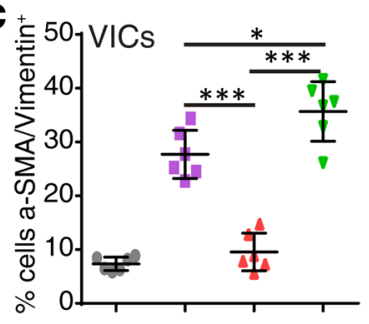

D

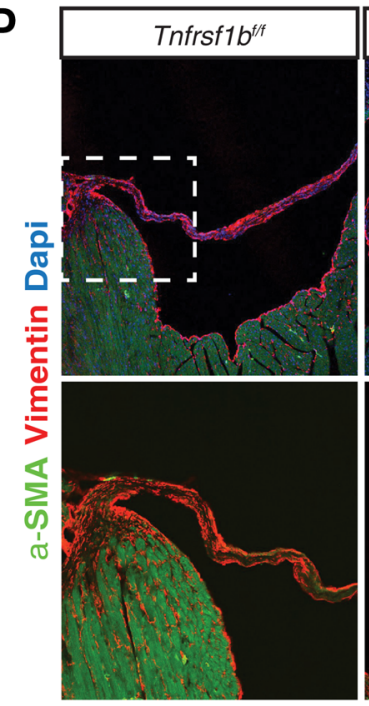

E

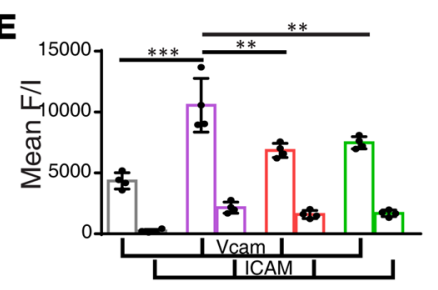

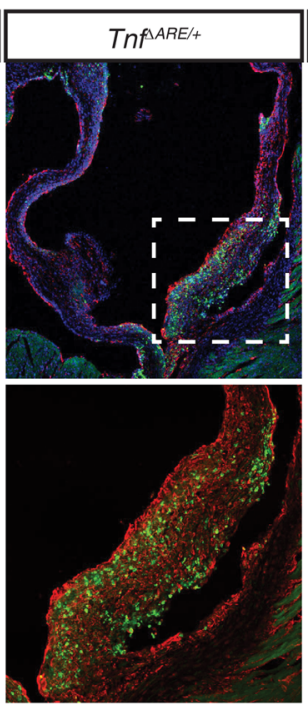

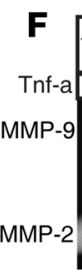

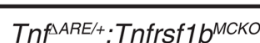

Tnf $\triangle A R E /+\cdot T n f r s f 1 b^{M C K O}$
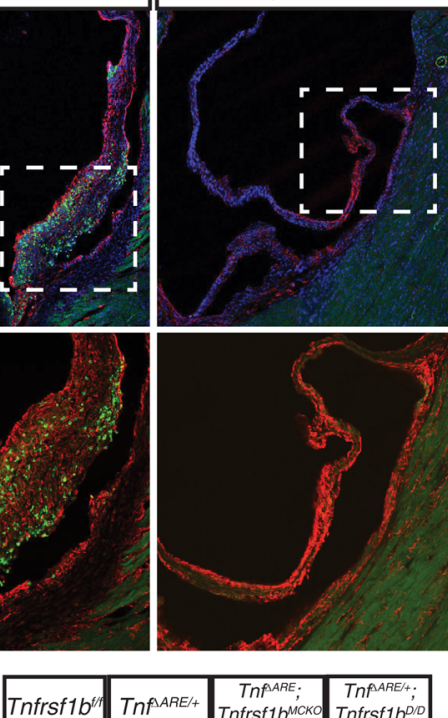
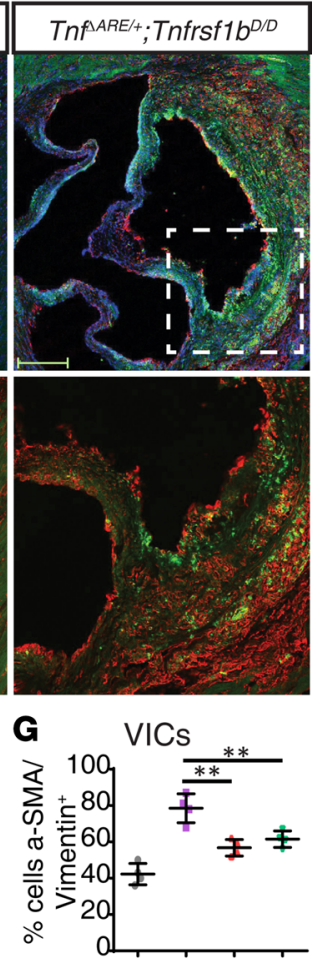

H

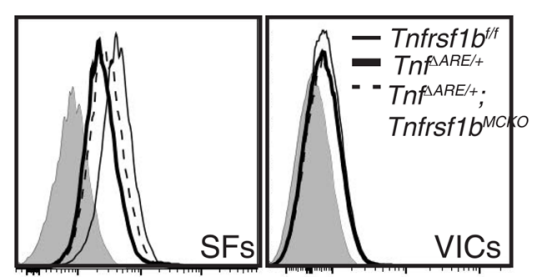

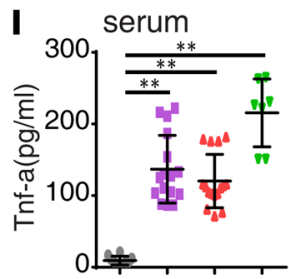

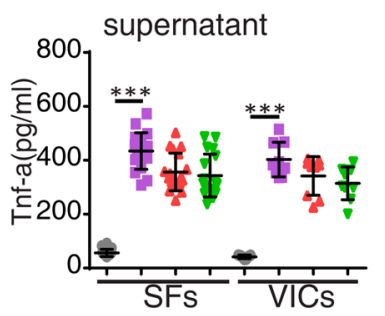

Figure 3. Mesenchymal Tnfr2 is required for fibroblast pathogenic activation in the ankle joint and the heart valve. Representative histogram for ICAM expression on crude joint cell preparations, performed by ICAM staining and FACS analysis (A). Graph bars include the quantitation of these data, depicting ICAM-1 $1^{+}$, synovial fibroblast (SF) \% over the gated $\mathrm{Cd} 45^{-}, \mathrm{Cd} 31^{-}$cell population (B), and double $\alpha$-smooth muscle actin $(\alpha-5 M A)^{+} /$vimentin $^{+}$valvular interstitial cells (VICs) \% in crude heart valve preparations over a similarly Cd45', Cd31- gated cell population (C) ( $n=6$ mice /genotype). Representative images of aortic valve leaflet sections stained for $\alpha$-SMA (green), vimentin (red), and DAPI (D). Bottom panels are $2 \times$ zoom-in of the indicated region of interests (ROIs) in the upper panels. Scale bar: $200 \mu \mathrm{m}$. (E) Mean fluorescence Intensity for Vcam-1 and ICAM expression quantified by immunostaining on cultured SFs and subsequent FACS analysis, ( $n=4$ mice/genotype). (F) Representative gelatin zymogram showing MMP-9 and MMP-2 secretion from cultured SFs, treated with $10 \mathrm{ng} /$ mI TNF as indicated. (G) Graph bars representing $\%$ of double $\alpha-\mathrm{SMA}^{+}$/ vimentin ${ }^{+}$cells in cultured VICs, quantified by staining and FACS analysis $(n=$ 4 mice/genotype). (H) Representative histograms for Tnfr1 expression on SFs and VICs performed by Tnfr1 staining and FACS analysis. (I) ELISA measurements of circulating soluble TNF in serum of 16-week-old mice ( $n=15$ mice/ genotype) and of secreted TNF in supernatants of cultured SFs and VICs ( $n=15$ mice/genotype for SFs and 9 mice/genotype for VICs) as indicated; the data are represented as means \pm SEM, in all the quantifications; and 1-way ANOVA and Bonferroni's multiple comparison tests were used to analyze groups for statistical significance $\left({ }^{*} P<\right.$ $\left.0.05,{ }^{* *} P<0.001,{ }^{* * *} P<0.0001\right)$.

of vimentin ${ }^{+} / \alpha-\mathrm{SMA}^{+}$cells compared with $\operatorname{Tnf}^{A R E /+}$ ones (Figure 3C). Moreover, immunocytochemistry performed on aortic valves revealed a substantial upregulation of double-vimentin ${ }^{+} / \alpha-\mathrm{SMA}^{+}$myofibroblast cells in the valve leaflets and roots of $\operatorname{Tnf}^{\text {fARE } /+}$ mice compared with WT, where VICs are in a quiescent state and $\alpha$-SMA expression is absent. Mesenchymal Tnfr 2 ablation led to complete suppression of $\alpha$-SMA expression and myofibroblast differentiation (Figure 3D), but in contrast Tnfr2 deficiency, that exacerbated valve stenosis in $T$ f $^{A A R E /+}$ background led to a marked increased number of $\alpha$-SMA ${ }^{+}$cells in the valve leaflets (Figure 3D). Thus, recapitulating the data we obtained from the FACS analysis, our results suggest that the increased proinflammatory milieu in the tissue, caused by total Tnfr2 deletion, affected the pathogenic activation status of fibroblasts independent of the mesenchymal Tnfr2 expression.

To further test this hypothesis, we isolated and cultured SFs and VICs from mice, and we analyzed disease-specific cell surface marker expression in all genotypes. Immunostaining and FACS analysis of SFs for the adhesion molecules Vcam-1 and ICAM, SF activation markers that are highly upregulated in culture, revealed that Vcam- 1 in cultured cells was significantly downregulated in both Tnf ${ }^{A R E /+} ; \operatorname{Tnfrs} 1 b^{\text {MCKO }}$ 


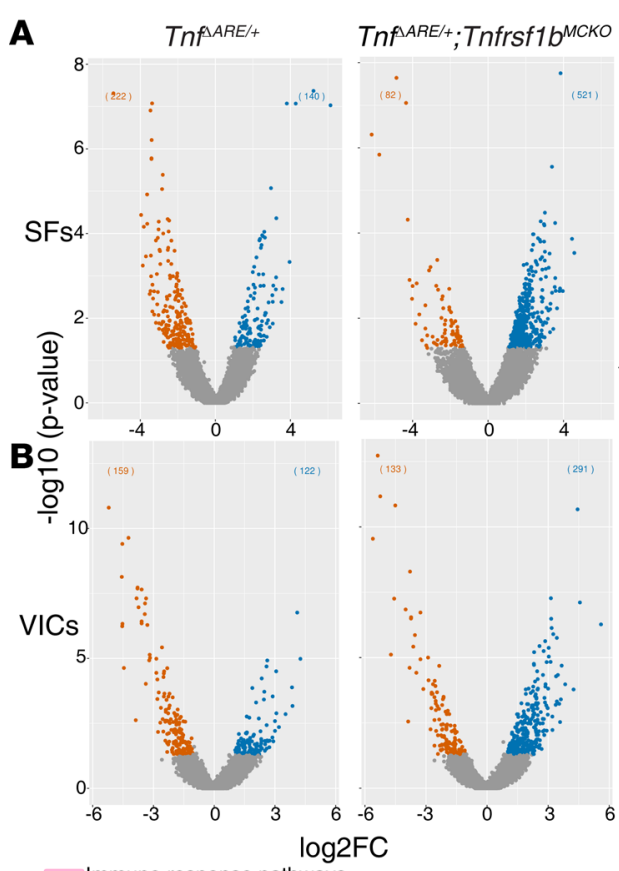

Immune response pathways

ECM, positive regulation of cell proliferation

cell adhesion
Cell metabolism related pathways

Disease specific pathways

\section{E significant Up significant Down SFs}

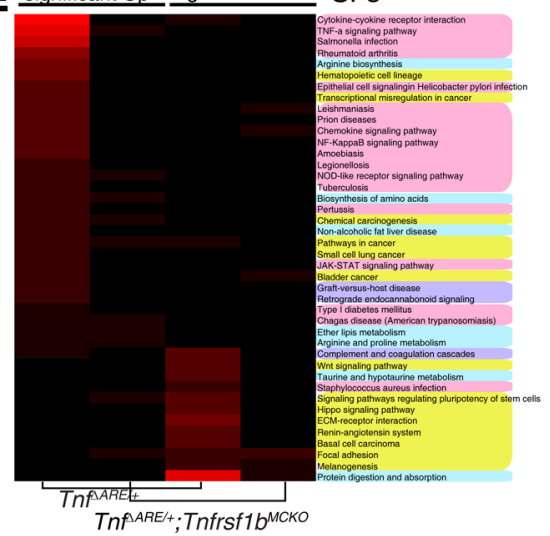

Tnf $\triangle A R E /+$;
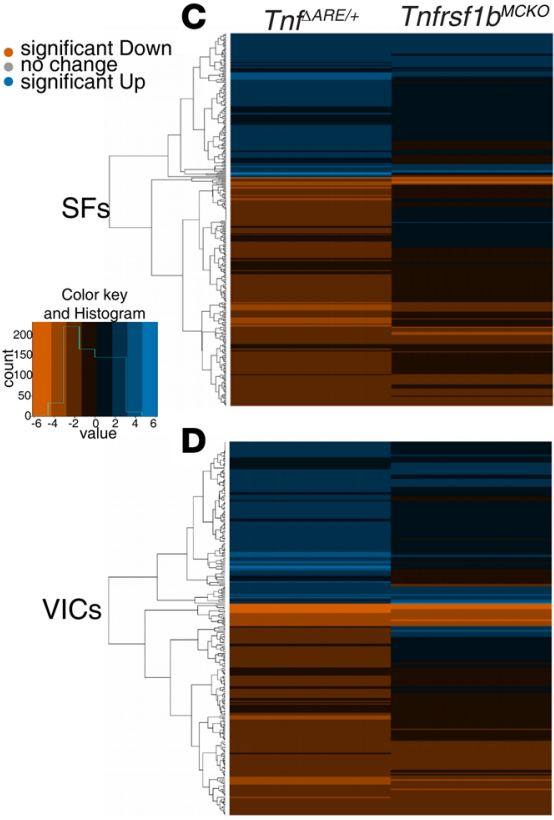

Color key

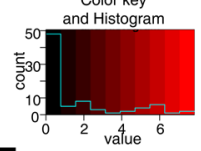

$F$

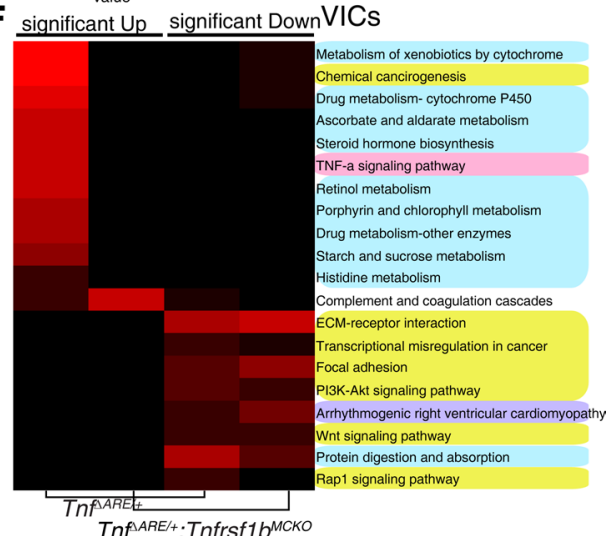

Figure 4. Deregulation of pathogenic SFs and VICs at the level of the transcriptome upon mesenchymal-specific deletion of Tnfr2 in the $\operatorname{Tnf}^{A A R E /+}$ mouse model. RNA-seq profiles of SFs and VICs from 8-week-old Tnf $f^{\triangle A R E /+}$, Tnf ${ }^{\triangle A R E /+} ;$ Tnfrsf $1 b$ ${ }^{M} \mathrm{CK}$, and WT controls showing the effect of mesenchymal Tnfrsfib depletion in the expression signature of $T \mathrm{Tf}^{\triangle A R E /+}$ in 3 distinct levels. Volcano plots depicting differentially expressed genes (DEGs) showing significantly upregulated (blue), no change (gray), and significantly downregulated (orange) in SFs (A) and VICs (B) in Tnf $f^{A A R E /+}$ compared with WT (first column) and in Tnf ${ }^{\triangle A R E /+} ;$ Tnfrsf1b ${ }^{\text {MCKO }}$ com- $^{-}$ pared with WT (second column). Heatmap showing fold change $\left(\log _{2}\right)$ expression values of significant DEGs in SFs (C) and VICs(D), of Tnf $\triangle A A R E /+$ (left column)

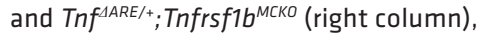
always in comparison with WT control. Functional analysis was performed on the DEGs and Kyoto Encyclopedia of Genes and Genomes (KEGG) pathways enriched in SFs (E) and VICs (F) were identified. KEGG pathways have been further categorized and color-coded into 4 clusters with distinct biological impact on cell function, as indicated.

and $T n f^{A R E /+} ; \operatorname{Tnfrs} f 1 b^{D / D}$ cells, whereas ICAM displayed a nonsignificant trend of downregulation in both genotypes compared with Tnf ${ }^{A A R E /+}$ levels (Figure 3E). These data indicate that, independent of the opposing in vivo effect that Tnfr2 in various cell types appears to have in arthritis, its deletion affected Vcam-1 and ICAM expression on isolated and cultured SFs in the same way. Vcam-1 has been implicated in the adherence of SFs to cartilage, whereby it mediates binding and potentiation of degrading enzymes such as matrix metalloproteinase (MMP) expression (10). MMP expression analysis by zymography showed that, similar to Vcam-1 expression, MMP-9 secretion and proteolytic activity against gelatin was strongly downregulated to WT levels in both Tnf ${ }^{A R E /+} ;$ Tnfrsf $1 b^{M C K O}$ and TnfARE/+$;$ Tnfrsf $1 b^{D / D}$ isolated SFs (Figure 3F). In addition, both Tnf ${ }^{A A R E /+}$; Tnfrsf $1 b^{M C K O}$ and Tnf ${ }^{A R E /+}$; Tnfrsf $1 b^{D / D}$ isolated VICs in culture presented significantly lower percentages of $\alpha$-SMA ${ }^{+}$cells, indicating a similar role for Tnfr2 in both cells types (Figure 3G) despite the opposing in vivo phenotypes. Up to now, pathogenic activation of fibroblasts was mainly attributed to the Tnfr1 signaling pathway (9). However, Tnfr1 expression on SF surface was not changed, as detected by immunocytochemistry and FACS analysis in both SFs and VICs (Figure 3H). In addition, circulating TNF as measured in the serum and supernatants of isolated SFs and VICs seemed to be unchanged as compared with the elevated levels measured in Tnf ${ }^{A R E /+}$ mice (Figure 3I), pointing to a more specific regulatory role of Tnfr2 downstream signaling in this process. Taken together, these data indicate that the failure of 
A
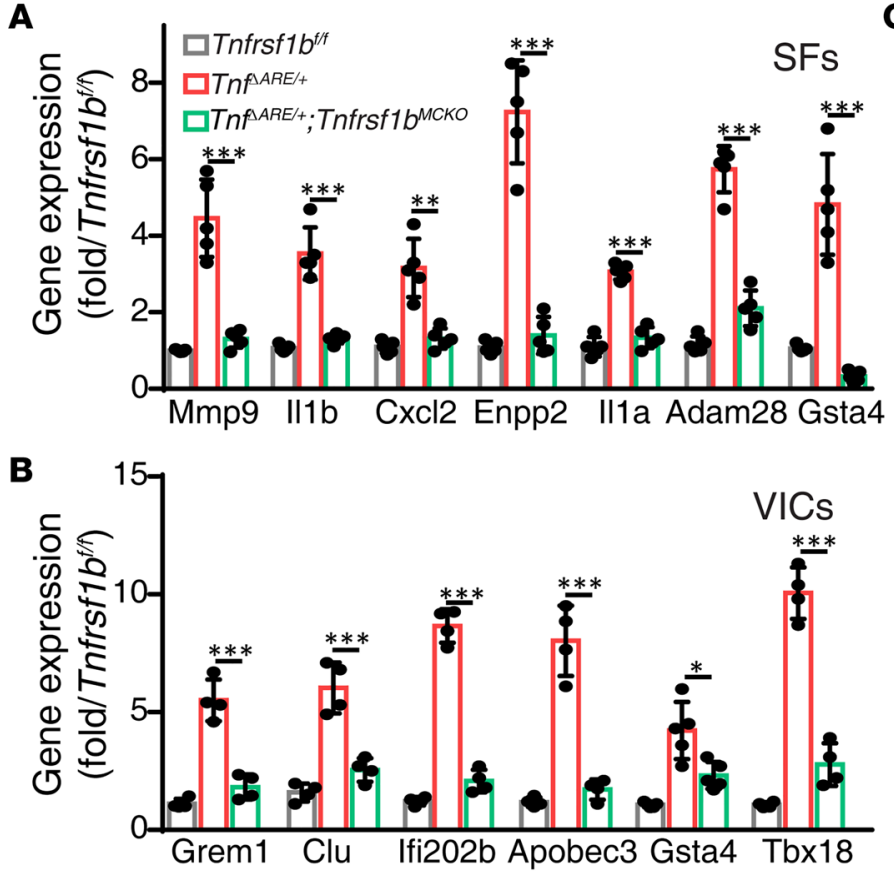

C
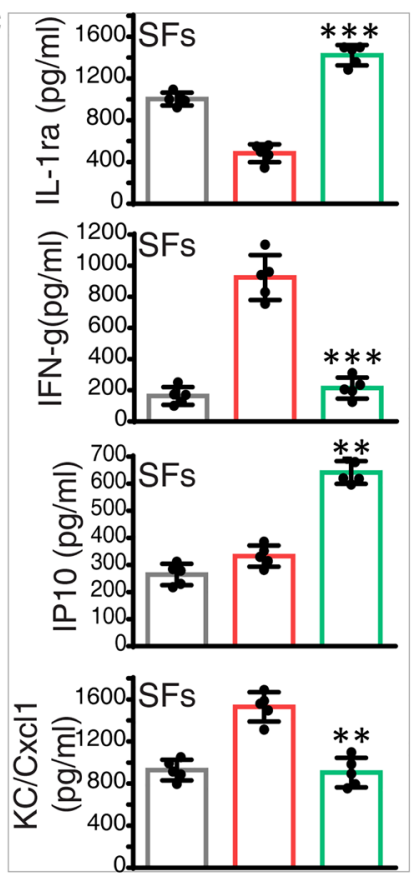

D

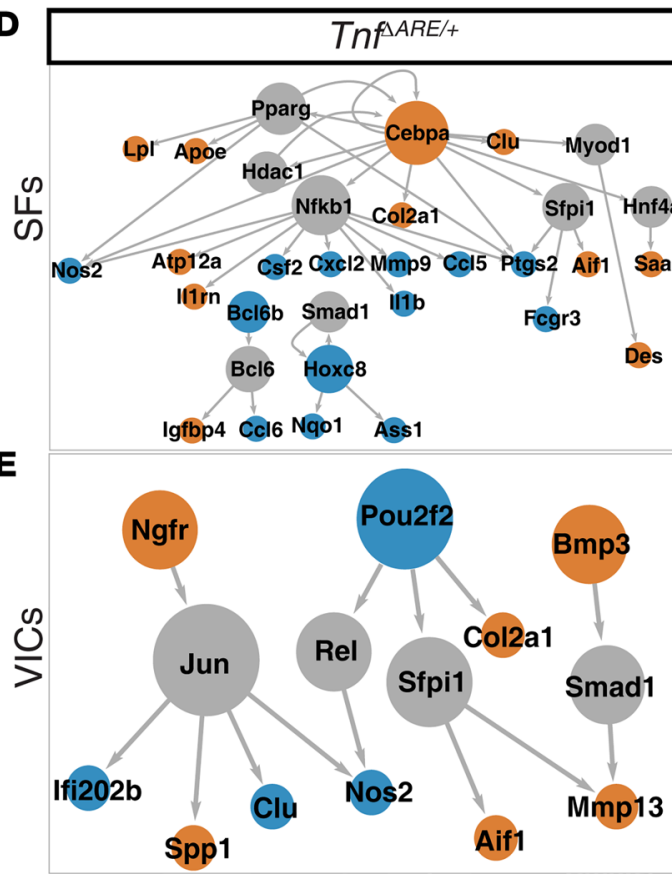

Tnf $^{\mathrm{AARE} /+} ; \operatorname{TnfrSf}_{1} \mathrm{~b}^{\mathrm{MCKO}}$

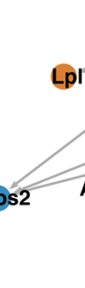

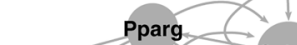

Cebpa Clu Myod1
Hdac1 I11'n Bci6b Smad1 ll1b

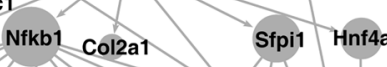

Atp12a Csf2 Cxcl2 Mmp9 CeI5 Ptgs2 Aif1 Saa1 Bcl6 Hoxc8 Fcgr3 Des Igfbp4 Ccl6 Nq01 Ass1

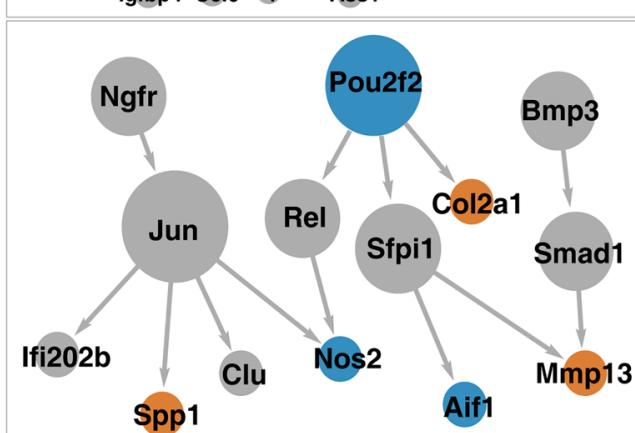

Figure 5. Pathogenic mesenchymal Tnfr2 signaling regulates the expression of molecular components in the PPAR- $\gamma$, NF- $\kappa B$, cell proliferation and disease-specific signaling pathways. Validation of selected deregulated genes as depicted by qPCR in SFs (A) and VICs

(B) isolated from 8-weekold Tnfrsf1 $1 b^{f / f}, T n f^{A A R E /+}$, and Tnf $^{\triangle A R E /+} ;$ Tnfrsf1b ${ }^{\text {MCKO }}$ mice. Values represent means $\pm S E M$; relative gene expression calculated to Tnfrsfib $b^{f / f}$ as $\Delta \Delta$ Ct values and normalized for each sample with Gapdh expression ( $n=5$ mice) genotype SFs, 4 mice/genotype for VICS). (C) ELISA measurements of secreted cytokines and chemokines from isolated SFs. Values represent means \pm SEM ( $n=5$ mice/genotype). All data sets were analyzed with 1-way ANOVA, and Bonferroni's multiple comparison tests were used to analyze groups for statistical significance $\left({ }^{*} P<0.05\right.$, ${ }^{* *} P<$ $\left.0.001,{ }^{* *} P<0.0001\right)$. Common transcriptional network in SFs (D) and VICs (E) of Tnf $f^{\triangle A R E /+}$ and Tnf $^{\triangle A R E /+} ;$ Tnfrsf1b ${ }^{\text {MCKO }}$ compared with WT, as identified with the regulatory network enrichment analysis tool. Circle size indicates node centrality, and color coding is identical to the one used in the volcano plots (Figure 4), indicating significant upregulation, no change, and significant downregulation.

$\operatorname{Tnf}^{\text {ARE } /+}$; Tnfrsf $1 b^{M C K O}$ and Tnf ${ }^{A A R E /+} ;$ Tnfrsf $1 b^{D / D}$ isolated SFs and VICs to become activated and to exert pathogenic functions arises as a direct effect of mesenchymal Tnfr2 ablation. In addition, mesenchymal Tnfr2 plays a dominant role in regulating these pathogenic cell activation pathways only when fibroblasts are in a cellular environment that expresses Tnfr2, since complete Tnfr2 deficiency leads to exacerbated fibroblast activation in both the joint and the heart valve. The nonmesenchymal cells contributing to the overall protective effects of Tnfr2 remain to be defined.

Tnfr2-ablated mesenchymal cells develop a normal phenotype with downregulated expression of pathogenic-related genes. To dissect the molecular mechanisms by which mesenchymal Tnfr2 regulates cell activation, we analyzed the transcriptome of highly pure short-term cultured joint and heart valve fibroblasts from WT, Tnf $f^{\mid A R E /+}$, and Tnf ${ }^{A A R E /+} ;$ Tnfrs $f 1 b^{M C K O}$ SFs and VICs using $3^{\prime}$ RNA sequencing (RNA-seq) (Supplemental Figure 8, A-D). We found a significant number of differentially expressed genes (DEGs) in $\operatorname{Tn} f^{A A R E /+}$ 
A

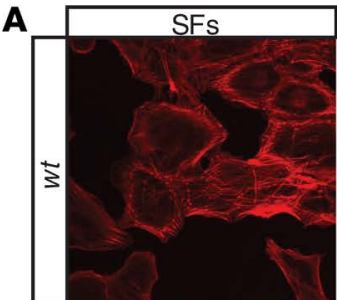

B

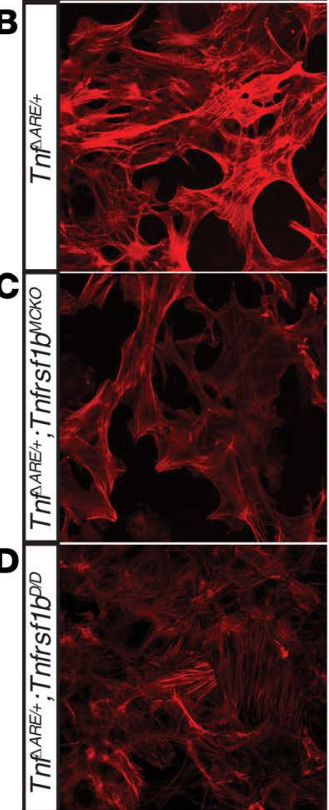

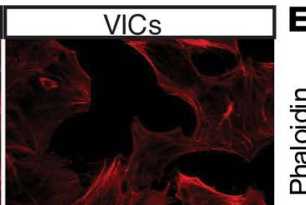

E

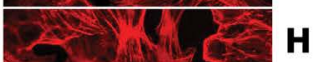

E

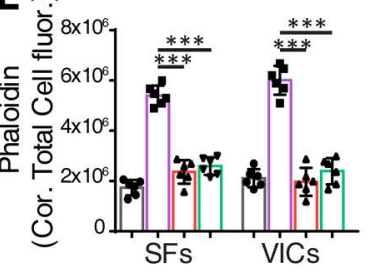

$\mathbf{F}$

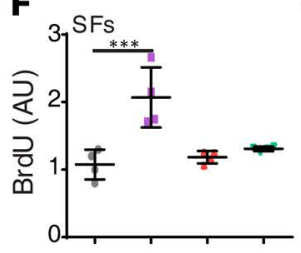

G

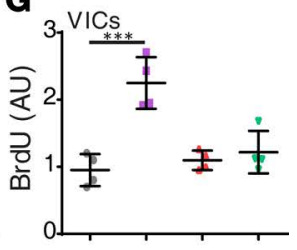

TnF $\mathrm{ARE} /+$

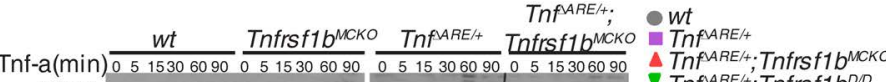

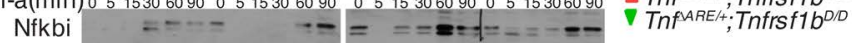

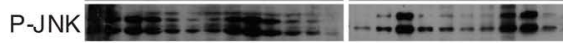

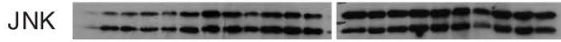
P-ERK =: : :

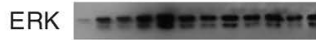
actin

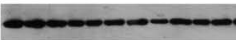

\section{.}

Tnf-a(min) $\overline{55153060} \overline{05153060} \overline{5} \overline{5153060} \overline{05153060}$ P-Akt - - - Akt - - - - - - - - - - - actin $-$
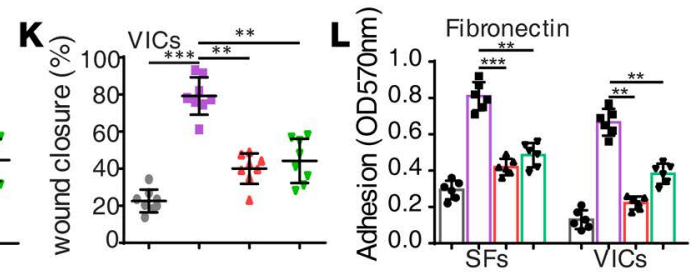

Figure 6. Tnfr2 regulates proinflammatory and proliferative gene expression in mesenchymal cells in the ankle joint and the heart valve in the Tnf ${ }^{1 A R E /+}$ mouse model, thus influencing their pathogenic function. Representative images of SFs and VICs isolated from 8-week-old WT (A), Tnf $\triangle A R E /+$ (B), $\operatorname{Tnf}^{\triangle A R E /+} ; \operatorname{TnfrSf} 1 b^{M C K O}(\mathbf{C})$, and $T n f^{\triangle A R E /+} ; \operatorname{TnfrS} f 1 b^{D / D}$ mice (D), stained with phalloidin for the cortical actin filaments. Scale bar: $100 \mu \mathrm{m}$. (E) Quantification of phalloidin staining; graph bars represent means of corrected total cell fluorescence quantification by Image) \pm SEM ( $n=30$ cells $/ 6$ mice/genotype). Cell proliferation quantification performed by ELISA colorimetric BrdU on isolated SFs (F) and VICs (C) ( $n=12$ wells/4 mice/genotype). Representative Western blots showing the expression of known downstream TNF signaling components, upon TNF stimulation time course as indicated $(\mathbf{H}$ and $\mathbf{I})(n=3)$. Wound closure measurements depicted as \% of the initial wound on confluent plates over 24-hour imaging of SFs (J) and VICs (K) as calculated by ImageJ ( $n=8$ mice/genotype). Adhesion quantification on fibronectin substrate quantified in both mesenchymal cell types by OD measurements of cells stained with crystal violet ( $n=6$ mice/genotype) (L). Quantitation for all data sets was performed with 1-way ANOVA and Bonferroni's multiple comparison tests were used to analyze groups for statistical significance $\left({ }^{* *} P<0.001,{ }^{* *} P<0.0001\right)$.

compared with WT cells with high reproducibility across samples; 140 genes were upregulated and 222 genes were downregulated in SFs, whereas 122 genes were upregulated and 159 genes were downregulated in VICs (Figure 4, A and B). Interestingly, a big proportion of the Tnf ${ }^{A A R E /+}$ DEGs ( $85 \%$ in SFs and $\sim 65 \%$ in VICs) were restored to WT levels upon depletion of Tnfr2, pointing to the pivotal role of Tnfr2 in establishing pathogenic signaling in both joint and aortic heart valve (Figure 4, C and D). Tnfrsfla expression is unchanged, indicating that Tnfr2 doesn't regulate Tnfr1 expression at the transcriptional level. Most of the deregulated genes belonged to pathways controlling cell homeostasis, such as NF-kB, PI3kAkt, pathogenic JAK/STAT pathway, proinflammatory nucleotide-binding oligomerization domain-like receptor (NOD receptor) signaling pathway adhesion, cell proliferation pathways, and extra-cellular matrix (ECM) interaction pathways as revealed by Kyoto Encyclopedia of Genes and Genomes (KEGG) pathway enrichment analysis and also highlighted with color coding (Figure 4, E and F). Moreover, the restoration of the DEGs observed upon Tnfr2 deletion is prominent at the pathway level, where almost all the KEGG pathways enriched in Tnf ${ }^{A R E /+}$ DEGs show no enrichment in Tnf ${ }^{A R E /+}$; Tnfrsf $1 b^{M C K O}$ DEGs, in both SFs and VICs. Notably, the deregulated genes included modulators of 2 key aspects of SFs and VICs pathogenic functions: inflammation and proliferation. Expression changes on selected genes of these pathways in both SFs and VICs were validated by quantitative PCR (qPCR) and perfectly correlated with the RNA-seq data (Figure 5, A and B). With respect to inflammation, Tnf ${ }^{A R E /+}$;Tnfrsf $1 b^{M C K O}$ SFs showed downregulation of 
A

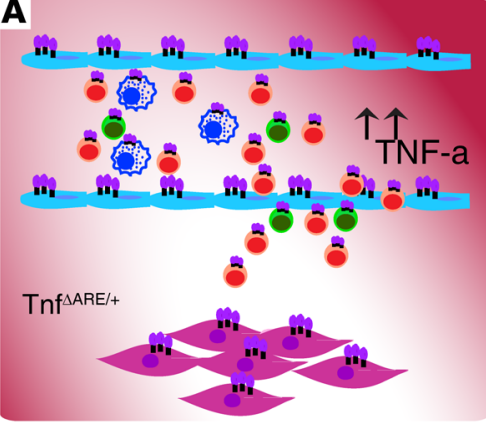

RA NHD comorbidity

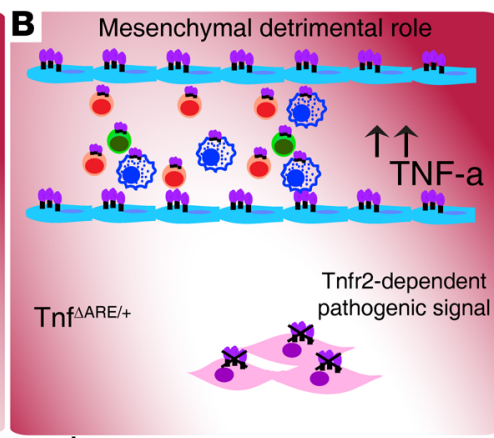

RA /VHD comorbidity

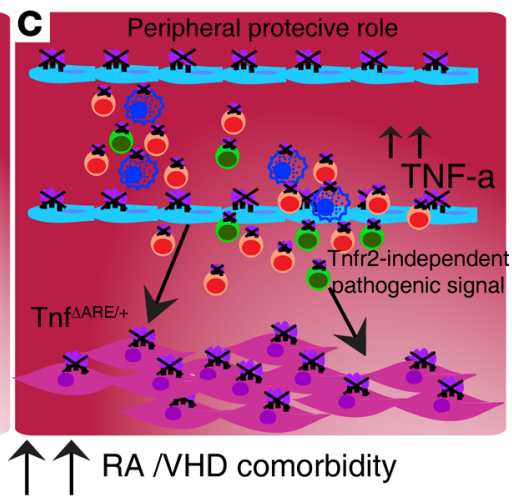

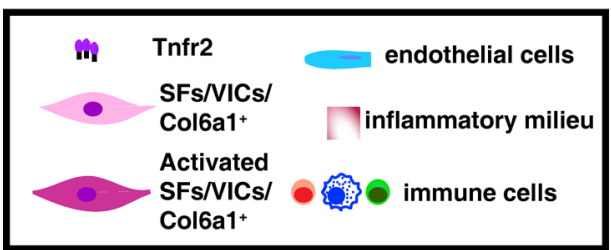

Figure 7. Model highlighting the pleiotropic functions of $\mathrm{Tnfr} 2 \mathrm{in}$ arthritis and valve stenosis comorbidity in the Tnf ${ }^{A A R E /+}$ mouse model. Tnf ${ }^{A A R E /+}$ mice develop polyarthritis and aortic valve stenosis that are mediated by TNF mesenchymal signaling and SF/VICs pathogenic activation (A). Col6a1-cre conditional Tnfr2 deletion revealed the detrimental role of mesenchymal Tnfr2 in both pathogenic processes (B). However, total deletion demonstrated the protective role that Tnfr2 signaling in immune and endothelial cells play. The highly inflammatory milieu created due to Tnfr2 deletion leads to exacerbated SFs and VICs activation through a Tnfr2-independent pathway (C). RA, rheumatoid arthritis; VHD, valvular heart disease.

proinflammatory cytokines and pathogenic MMPs, as quantified also by ELISA assays (Figure 5C), and upregulation of antiinflammatory cytokines such as IP10 and IL-1ra (Figure 5C). With respect to proliferation, Tnf ${ }^{A R E /+}$;Tnfrsfl ${ }^{M C K O}$ SFs showed a significant decrease of oncogenes such as Bcl6, Grem1, and Tac1, which are found to be upregulated in $\operatorname{Tnf}^{A R E} /+\mathrm{SFs}$. Furthermore, analysis of transcriptional regulatory networks, as identified with the regulatory network enrichment analysis (RNEA) tool (51), orchestrating the phenotype in TnfARE/+ SFs and VICs, indicated that the majority of pathogenic pathways that are activated downstream of TNF are also restored to WT levels and, thus, silenced in TnfARE/+ $;$ Tnfrsf1 $1 b^{\text {MCKO }}$ SFs (Figure 5, D and E). This indicates that, in SFs, transcriptional regulation of pathogenic markers such as Mmp 9, Il1, Illrn, and Cxcl2 not only depend on the direct Tnfr1 activation, but also on the auxiliary presence of Tnfr2, pointing to an additive signaling of Tnfr1 and Tnfr2 in SFs. However, in VICs, KEGG analysis pathways indicated similarities between the downstream targets of Tnfr2 in the joint and the heart valve mesenchymal cells, including metabolic and cell proliferation pathways. However, there seems to be no regulation at the transcriptional level of proinflammatory cytokines in this cell type, which could also explain the lack of immune cell infiltration in the aortic valve leaflets of TnfARE/+ mice (Figure 4F). However, ELISA quantification of pro- and antiinflammatory cytokines secreted by VICs demonstrated that Tnfr2 also exerts a regulatory role in their production and secretion ex vivo.

Collectively, these data indicate that, in the Tnf ${ }^{A R E} /++$ mouse model, SFs and VICs express activatory and pathogenic profiles, whereas mesenchymal deletion of Tnfr2 in the joint and the heart valve leaflets results in reprogramming of these cells to a quiescent nonpathogenic state.

Cultured SFs and VICs display Tnfr2-dependent proinflammatory activation, hyperproliferation, and migratory functions. To assess mesenchymal Tnfr2 functions as identified by RNA-seq, we performed cell assays on isolated SFs and VICs. Interestingly, both SFs and VICs isolated from WT mice display a multipolar elongated cell shape and low levels of F-actin as observed by phalloidin staining (Figure 6A). However, activated TnfARE/+ SFs and VICs were, in their majority, bipolar and showed significantly higher levels of phalloidin staining due to the more complex and denser actin filament networks in the cells (Figure 6B). This effect of TNF on the fibroblast actin network has already been reported for SFs $(52,53)$, but it is the first time to our knowledge that its role is also characterized in VICs. Interestingly though, both SFs and VICs - upon Tnfr2 ablation - are WT-like and are in mostly multipolar, with low levels of membrane F-actin, independent of whether the cells were isolated from Tnf $f^{A R E /+} ;$ Tnfrsf $1 b^{M C K O}$ or Tnf ${ }^{A R E /+} ;$ Tnfrsf $1 b^{D / D}$ mice (Figure 6, C and D). Phalloidin staining quantifications revealed that $T n f^{A R E /+} ; \operatorname{Tnfrs} f 1 b^{M C K O}$ cells exerted significantly lower F-actin polymerization 
(Figure 6E). Moreover, we have shown that, in the Tnf ${ }^{A R E /+}$ model, SFs hyperproliferate and form the destructive pannus that propagates the disease $(7,8)$. Similarly, we also observed a marked increase of the Col6a1 ${ }^{+}$ cells in the valve leaflets of TnfARE/+ mice, indicating that increased VIC proliferation could also be central to valve stenosis. Indeed, a 4-hour BrdU incubation and subsequent staining on isolated SFs and VICs showed that Tnf $f^{A R E /+}$ cells display a 2- to 3-fold increase in proliferation as compared with control Tnfrsf $1 b^{f / f}$ cells, and both SFs and VICs lacking Tnfr2- Tnf ${ }^{A R E /+} ;$ Tnfrs $f 1 b^{M C K O}$ and Tnf ${ }^{A R E /+} ;$ Tnfrsf $1 b^{D / D}$ displayed WT-like levels of proliferation (Figure 6, F and G). These experiments indicated that Tnfr2 modifies the proliferation ratio of SFs in the joint and VICs in the heart valves and, thus, influences both pathologies. Furthermore, these data provide a functional validation of the RNA-seq data that revealed a regulation of proliferation-related genes at the transcriptional level downstream of Tnfr2. In addition, analysis of the signaling events downstream of TNF showed that TNF-canonical signaling in Tnf ${ }^{A R E /+}$; Tnfrs $f 1 b^{M C K O}$ SFs were comparable with WT control, since JNK and ERK kinases displayed similar phosphorylation kinetics and nuclear factor of $\kappa$ light polypeptide gene enhancer in B cells inhibitor, $\alpha$ (Nfkbi) showed physiological degradation (Figure $6 \mathrm{H}$ and Supplemental Figure 9, A-C). In contrast, Akt kinase, which belongs to the noncanonical TNF signaling pathway and is associated with positive regulation of cell proliferation and cancer, fails to become phosphorylated, pointing to another level of Tnfr2-mediated cell proliferation regulation downstream of TNF that associates with the PI3K-Akt signaling pathway (Figure 6I and Supplemental Figure 9D), similar to what has been observed in cancer cells $(54,55)$.

Another pathogenic feature of the TnfARE/+ SFs is the increased migration potential that could be explained by changes that local TNF secretion brings in actin cytoskeleton dynamics, increased MMPs secretion, and JNK-downstream signaling upregulation as shown for tumor cell migration and invasion $(56,57)$. To examine the role of Tnfr2 in regulating cell migration in SFs and VICs, we performed and quantified wound closure assays in the presence of mitosis inhibitor mitomycin. Twenty-four-hour timelapse imaging revealed that Tnf ${ }^{A R E /+}$ SFs and VICs displayed $70 \%-80 \%$ closure of the wound performed

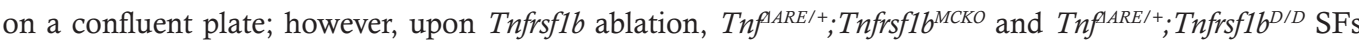
and VICs showed only $40 \%-45 \%$ closure in the same time frame, indicating that Tnfr2 signaling regulates cell migration in mesenchymal cells and, thus, pathogeneity (Figure 6, J and K). Last, increased substrate adhesion has been shown to be causal to the initiation of cartilage and bone destruction during arthritis pathogenesis. Surprisingly, despite the fact that TnfARE/+ $;$ Tnfrsf $1 b^{M C K O}$ and Tnf ${ }^{A R E /+} ;$ Tnfrsf $1 b^{D / D}$ SFs and VICs display typical TNF signaling, the adhesion capacity of both cell types on a fibronectin substrate was reduced by almost 50\% compared with the levels measured for Tnf $f^{\text {ARE } /+}$ cells (Figure 6L). Taken together, these data functionally confirm the pathways we identified during the transcriptomic analysis of pathogenic Tnf ${ }^{A A R E /+}$ and Tnf ${ }^{A R E /+}$; Tnfrsf $1 b^{M C K O}$ mesenchymal cells, which — for the first time to our knowledge - identify the role of Tnfr2 in directly regulating pathogenic proinflammatory signals, proliferation rates, and the migration potential of the cells during arthritis and heart valve stenosis pathogenesis.

\section{Discussion}

In the present study, we uncover the pleiotropic functions of joint and heart mesenchymal Tnfr2 versus other cell types in modeled arthritis and heart valve stenosis comorbidity. We demonstrate that, in the presence of systemic Tnfr2, mesenchymal Tnfr2 is detrimental for both arthritis and valve stenosis pathophysiologies by providing proliferative and proinflammatory signals that drive their chronic pathogenic activation. In contrast, nonmesenchymal Tnfr2 signaling appears dominantly protective for both pathologies (Figure 7).

Until now, Tnfr2 - unlike Tnfr1 — was believed to display an inducible expression limited mainly to immune, endothelial, neuronal, microglia, oligodendrocytes, thymocytes, and mesenchymal stem cells (30, 35, 36, 58-60). Unlike Tnfr1, which exerts mainly proapoptotic functions, Tnfr2 cannot recruit the adaptor molecule TRADD because it does not have a death domain in its intracellular part. However, Tnfr2 can recruit TNF receptor associated factor 2 (TRAF2) and thereby activate the NFkB pathway and MAPKs. Tnfr2 can also sensitize cells to apoptosis by competing with Tnfr1 for TRAF2 and baculoviral iap repeat containing (cIAP) molecules, which are important for formation of Complex-I. This might lead to a decrease in the expression of survival genes and allow Complex-II to induce apoptosis (61). Paradoxically, though, the most common effect of Tnfr2 signaling is proliferation and prosurvival functions, probably caused by differential NF-kB-dependent transcriptional repertoire downstream of the 2 receptors. From a physiological point of view, Tnfr2 signaling exerts immunomodulatory functions, remyelination, and neuronal survival; however, these Tnfr2 functions are not involved in disease pathophysiology in the 
TnfARE/+ mouse model of polyarthritis (7). Total $\mathrm{KO}$ of $\mathrm{Tnfr} 2$ in the Tnf ${ }^{A R E /+}$ mouse model exacerbated disease progression, pointing to an antiinflammatory protective role of systemic Tnfr2 signaling in arthritis. This could be explained by its function on stromal and peripheral cells forming a protective antiinflammatory barrier. We went further to test whether Tnfr2 was also expressed and potentially playing a role in the mesenchymal cells lining the joint and the heart valve in arthritis and valve stenosis pathogenesis in $T_{n} f^{A R E /+}$ mice. Interestingly, we detected strong Tnfr2 expression in both SFs in the ankle joint and mesenchymal cells in the heart tissue, including VICs in the valves. Because SFs mediate arthritis pathology in the $\operatorname{Tnf}^{\text {ARE } /+}$ mouse model, we hypothesized that mesenchymal Tnfr2 could be the executor, at least in part, of TNF-mediated detrimental functions in vivo.

To support this hypothesis, we first investigated whether Tnf ${ }^{A A R E /+}$ mice developed pathology and examined the role of mesenchymal origin VICs in this. We demonstrate that the reported gross thickening of the aortic valve leaflets in the $\operatorname{Tnf}^{A A R E /+}$ mouse model is the result of TNF-mediated hyperproliferation of resident mesenchymal origin cells, increased collagen deposition, and marked fibrosis. TNF signaling in the TnfARE/+ model drives valve mesenchymal cell activation and myofibroblast differentiation, rather than lymphocyte infiltration and inflammation, as previously reported in other models (20, 21). Interestingly, mice displaying cardiomyocyte-specific overexpression of TNF develop myocarditis and cardiomegaly (19, 62). Moreover, it is noteworthy that $\operatorname{Tnf}^{A R E /+}$ mice in this study were maintained in C57BL/6 background, whereas BPSM1 mutant mice - which, similar to the Tnf ${ }^{A A R E /+}$, display strong overexpression of mouse TNF, severe valvulitis, and immune cell infiltration - were kept on BALB/c genetic background (21). However, recent studies revealed that BALB/c mice develop spontaneous cardiac inflammation, lymphocyte infiltration, and calcinosis; thus, the BALB/c-related phenotypes may be due to the specific predispositions of this background (63).

Capitalizing on the mesenchymal-specific origin of both SFs and VICs, the Col6a1-cre mouse line allowed us to target mesenchymal Tnfr2 expression. Total deletion of Tnfr2 in TnfARE/+ $;$ Tnfrsf $1 b^{D / D}$ mice resulted in worsening of the clinical outcome for both arthritis and valve stenosis, earlier onset, and even alteration of the pathologies since marked immune cell infiltration was observed in both sites compared with TnflARE/t. Unexpectedly, but in support of the mesenchymal hypothesis $(10,11,39,64)$, $T_{\text {Tnf }}^{\text {IARE } /+}$; Tnfrsf $1 b^{M C K O}$ mice showed the opposite; mesenchymal Tnfr2 ablation protected the mice from arthritis and valve stenosis because of the inability of SFs in the joint and VICs in the heart valve to acquire a pathogenic chronically activated status.

To gain insight into the molecular mechanisms of mesenchymal Tnfr2-dependent regulation of arthritis and valve disease comorbid manifestation, we performed RNA-seq analysis of SFs and VICs isolated from Tnf ${ }^{A R E /+}$; Tnfrsf $1 b^{\text {MCKO }}$ and TnfARE/+ mice at disease onset. Three key findings arose from this analysis. First, Tnfr2 ablation causes silencing of a large majority of genes that display deregulated expression in $\operatorname{Tn} f^{A R E /+}$ mice in both SFs and VICs. The impaired ability of Tnfr2-deficient cells to respond to pathogenic levels of TNF and cause arthritis and valve stenosis is reflected in the silenced expression of a large number of pathogenic, deregulated genes. These include genes involved in extracellular matrix receptor interaction, a fundamental feature of pathogenic fibroblasts, such as integrins, integrin-binding molecules, syndecan1, focal adhesion complex proteins, and modulators, but also a large number of collagen, such as Col2a1, Col6a2, Col14, Col15a1, and Coll1a1. Specifically, in SFs, TNFR2 regulates expression of arthritis-specific genes that are involved in ECM, cartilage, and bone destruction, such as Mmp8, Mmp9, Adam28, other disease-related genes — including Tac1, Traf1, and numerous canonical Wnt signaling pathway molecules. Likewise, in VICs, Tnfr2 regulates valve stenosis disease-related genes that were deregulated in $\mathrm{Tnf}^{\mathrm{ARE} /+}$ mice, such as Peg3, Procr, Tgfb signaling components, Bmp3, Adamts1, and Agtr1.

The second key finding of the RNA-seq functional analysis is that, in Tnf ${ }^{A R E /+}$, Tnfr2-ablated SFs and VICs express WT levels of genes regulating proliferation, cell migration, and cytoskeleton dynamics pathways that are highly upregulated in Tnf ${ }^{A R E /+}$ cells. One example is the Grem1 oncogene $(65,66)$, which is strongly upregulated in TnfARE/+ SFs and VICs and correlates with pannus formation in the joint and valve stenosis in the heart, suggesting that this could underlie mesenchymal cell hyperproliferation downstream of Tnfr2 and be detrimental for both pathologies. Similar, Rhoa GAPs, profilin1 (Pfn1), and Rac1 were downregulated in cells lacking Tnfr2. The pathways and genes that we mentioned until now are commonly regulated by Tnfr2 in both SFs and VICs. A third key finding of our analysis is that Tnf $f^{A R E /+}$ SFs display a proinflammatory and catabolic phenotype correlating with arthritis pathophysiology. Moreover, Tnfr2-deficient SFs display downregulated proinflammatory chemokine secretion, including I11a, Cxc12, and Cc16, that could explain 
the decreased influx of immune cells in TnfARE/+ Tnfrsf $1 b^{M C K O}$ mouse joints. In contrast, VICs do not display similar phenotypes. Very few antiinflammatory genes are downregulated in $\operatorname{Tn} f^{A R E /+}$ VICs, thereby reflecting the anabolic nature of valve stenosis characterized mainly by hyperproliferation and fibrosis.

For a full understanding of Tnfr2 function in arthritis and aortic valve stenosis, we analyzed the cellular behavior of SFs and VICs ex vivo. Mesenchymal cells of Tnf ${ }^{A R E /+}$; Tnfrsf $1 b^{D / D}$ don't express the receptor, just like TnfARE/+ $T$ Tnfrsflb ${ }^{\text {MCKO }}$ ones; however, unexpectedly, in the ankle joint and the valve cells developed a chronic, fully activated phenotype and significantly higher immune cell infiltrations compared with Tnf $f^{\text {ARE } /+}$. However, isolated SFs and VICs behaved identically to Tnf ${ }^{\text {ARE } /+} ;$ Tnfrsf $1 b^{\text {MCKO }}$. Tnfr2 is strongly expressed on immune cells, where it regulates 2 key steps of cell activation, expansion and differentiation $(30,36,67)$, but also in endothelial cells, where it regulates angiogenesis and endothelial barrier-related functions $(35,68)$. Interestingly, proliferation, expansion, and ECM regulation signaling pathways that have been shown to be regulated by Tnfr2 in immune and endothelial cell types are also regulated by Tnfr2 in SFs and VICs, since we found them suppressed in mesenchymal Tnfr2-ablated TnfARE/+ mice. These data suggest that the outcome of the phenotype may depend more on the cell-specific responses to such signals rather than activation of distinct signaling pathways in each cell type. Indeed, Tnfr2-dependent signals in nonmesenchymal cells in Tnf ${ }^{A R E /+}$ mice suppress inflammation and pathogenic activation SFs and VICs, since, in their absence, the joints and heart valve are more vulnerable to immune-inflammatory attack from the periphery. As arthritis and valve stenosis progress in Tnf ${ }^{A R E /+} ; \operatorname{Tnfrs} f 1 b^{\mathrm{D} / \mathrm{D}}$ mice, the clinical profiles of both pathologies are exacerbated compared with $\operatorname{Tnf}^{A R E /+}$, and this may be due to more immune cell infiltration in the joint and the aortic valve overcoming the antiinflammatory barrier set by nonmesenchymal Tnfr2-expressing peripheral cells, possibly caused by impaired immune and endothelial cell function. Nevertheless, our data highlight that the peripheral protective role of Tnfr2 in the early stage of disease is underscored by the detrimental mesenchymal role of Tnfr2, which seems to play a dominant role exclusively in the presence of peripheral Tnfr2 expression.

Finally, our work further highlights the complexity of TNF/Tnfr2 functions in arthritis and CV comorbid diseases depending on the location in mesenchymal or peripheral myeloid and endothelial cells.

Thus, total ablation of Tnfr2 leads to increased immune cell infiltration and exacerbation of the proinflammatory milieu in both the ankle joint and the heart valve, possibly caused by impaired immune and endothelial cell function.

Taken together, our data also underline the dichotomy of Tnfr2 signaling in pathophysiology. On the one hand, mesenchymal Tnfr2 mediates a detrimental role for disease initiation and progression. However, on the other hand, total Tnfr2 deficiency exacerbates the disease, pointing to a nonmesenchymal protective role and indicating in parallel that systemic receptor ablation leads to the activation of a Tnfr2-independent pathogenic mesenchymal signal (Figure 7). In this context, Tnfr1, which is still expressed on SFs and VICs, seems to be sufficient to overly activate fibroblasts and worsen the clinical outcome of arthritis and aortic valve stenosis in the absence of immune and endothelial Tnfr2 signaling.

\section{Methods}

Mice. All mice were bred and maintained on C57BL/6J and on mixed C57BL/6J $\times 129 \mathrm{~S} 6$ or C57BL/6 $\times$ CBA genetic backgrounds in the animal facilities of the BSRC Alexander Fleming under specific pathogen-free conditions (Charles River Laboratories; The Jackson Laboratory). Adult (2-20 months) female and male mice were used in this study. The conditional Tnfrsflb, Tnfrsflbff mouse strain, was generated using standard gene-targeting techniques. In brief, a targeting vector was generated to induce homologous recombination in ES cells, with exon 2 flanked by loxP sites, which - upon recombination - would result in a frame shift mutation. Upstream of the first loxP site, a flipped neo cassette was inserted to allow for antibiotic-resistance selection, and flanking sequences of 2.8 and $3.6 \mathrm{~kb} \mathrm{5'}$ and $3^{\prime}$ completed the vector, fulfilling the homologous recombination requirements. All sequences of the mouse Tnfrsflb gene were derived by PCR amplification from the respective BAC clone. All exons have been sequenced, and the integrity of the sequence was confirmed. The vector was introduced in Bruce 4 ES cells (MilliporeSigma), and clones, having successfully recombined, were used for the generation of chimaeras. Chimeric mice were crossed to C57BL/6J mice, and offspring with germline transmission of the mutated allele were used for expansion. The mice were crossed to FLP Deleter mice for the removal of the neo cassette. Then, Tnfrsfl $1 b^{f f}$ mice were crossed either with Cre Deleter mice for the generation of Tnfrsflb-complete KO mice, designated as Tnfrsf $1 b^{D / D}$ mice, or with Col6a1-cre mice for the generation of mice with specific deletion of TNFR2 in the mesenchymal compartment, Tnfrsf $1 b^{M C K O}$. The generation of Tnf ${ }^{A R E /+}$, 
transgenic Col6a1-cre, and mutant mice carrying a conditional gain-of-function allele for Tnfrsfla (Tnfrsfla flxneo mice) have been previously described $(9,69)$. ROSA26 $6^{f x /+}$ mice was purchased from The Jackson Laboratory.

Histopathology, IHC and immunofluorescence. Histological stainings, H\&E, Masson Trichrome, and Toluidin blue, were performed on paraffin heart valve and ankle joint sections as previously described (9). For IHC, paraffin or cryosections were probed with antibodies against Col6a1 (Abcam, ab6588), CD3 (Abcam, ab16669, clone Sp7), B220 (BD Pharmingen, 553084, clone RA3-6B2), F4/80 (AbD Serotec, MCA497, clone 0215), Neutrophil (Abcam, ab2557, clone NIMP-R14), vimentin (A647-conjugated, Abcam, ab194719, clone EPR3776), Tnfr2 (D2, Santa Cruz Biotechnology Inc., sc8041), and $\alpha$-SMA (MilliporeSigma, A5228, clone 1A4). Alexa-Fluor 647-conjugated secondary antibodies (anti-rabbit, A21244, 1834794; anti-rat, A21247, 1719171; anti-mouse:, A21235, 1868116; and anti-hamster, A21451, 1572558, Invitrogen), biotinylated secondary antibodies (anti-rat, BA-9400, and anti-rabbit, BA-1000, Vector Laboratories), the Vectastain ABC kit (PK-6100, Vector Laboratories), and the Vectastain ImmPACT DAB kit (SK-4105, Vector Laboratories) were used for detection and signal amplification. Images were acquired with a TCS SP8X White Light Laser confocal microscope (Leica) and with an Eclipse E800 (Nikon) microscope equipped with a Dxm1200F camera (Nikon). Imaging analysis and quantifications were performed with ImageJ/Fiji software (NIH).

Echocardiographic and electrocardiographic examination (ECGS). Echocardiography assessment was performed at the German Mouse Clinic (Munich, Germany) on conscious animals. Left ventricular function was evaluated with transthoracic echocardiography using a Vevo 2100 Imaging System (Visual Sonics). Briefly, left ventricular parasternal short- and long-axis views were obtained in B-mode imaging, and left ventricular parasternal short-axis views were obtained in M-mode imaging at the papillary muscle level. The short-axis M-mode images were used to measure left ventricular end-diastolic internal diameter (LVEDD), left ventricular end-systolic internal diameter (LVESD), diastolic and systolic septal wall thickness (interventricular septum; IVS), and diastolic and systolic posterior wall thickness (LV posterior wall thickness; LVPW) in 3 consecutive beats according to the American Society of Echocardiography. Fractional shortening (FS) was calculated as FS\% $=[($ LVEDD - LVESD)/LVEDD $] \times 100$ and ejection fraction $(\mathrm{EF})$ was calculated as $\mathrm{EF} \%=[(\mathrm{LVvolD}-\mathrm{LVvolS}) / \mathrm{LVvolD}] \times 100$ with $\mathrm{LVvol}=[7 /(2.4+$ LVID) $\times$ LVID3], where LVvolD represents left ventricular diastolic volume and LVvolS represents left ventricular systolic volume. Cardiac output (CO) represents the volume of blood pumped by the heart per minute, was standardized relatively to the body weight of each mouse, and was measured by Doppler flow velocity measurements.

ECGs were recorded by ECGenie (Mouse Specifics Inc.) and analyzed using e-Mouse software (Mouse Specifics Inc.). The cardiac electrical activity was detected through 3 of the animals' paws, and for each animal, intervals and amplitudes were evaluated from continuous recordings of at least 15 ECG signals. $\mathrm{HR}$ and $\mathrm{HR}$ variability was subsequently calculated as the mean of the differences between sequential HRs as calculated by the e-Mouse software.

Cell isolation and cell culture assays. SF cultures were performed as previously described (52). VIC cultures were established by enzymatic treatment of isolated aortic valve leaflets from 8-week-old mice, as previously reported (70). The purity of VIC cultures was determined by flow cytometric detection of Cd90, vimentin, $\alpha$-SMA, CD29, ICAM-1, Vcam, podoplanin/Gp38, and Cd45/Cd31 expression. At passages 3-4, cells were treated with $10 \mathrm{ng} / \mathrm{ml}$ TNF (provided by C. Libert, Ghent University, Belgium) or $1 \mu \mathrm{g} / \mathrm{ml}$ LPS (MilliporeSigma) for the indicated time points (zymography and signaling experiments). Cell proliferation of SFs and VICs was quantified using a Colorimetric immunoassay kit (Roche Diagnostics) based on the measurement of BrdU incorporation during DNA synthesis according to manufacturer's instructions. The wound closure assay of SFs and VICs was performed as previously described (71) and was observed by time-lapse imaging over 24 hours in stable conditions $\left(37^{\circ} \mathrm{C}, 5 \% \mathrm{CO}_{2}\right)$ in a Zeiss Axio Observer $\mathrm{Z1}$ microscope. Quantifications were performed with ImageJ/Fiji (NIH). For qPCR and target verification, total RNA was isolated from cultured SFs and VICs using the TRIZOL reagent (Invitrogen, 15596026). Complementary DNA was prepared with the Reverse Transcription system (Promega) according to the manufacturer's instructions. qPCR was performed on a CFX96 Real-Time System (Bio-Rad) with the Platinum SYBR Green master mix (Invitrogen). Gene expression fold change was subsequently calculated by $\Delta \Delta \mathrm{Ct}$ analysis using Gapdh as control Ct values for each gene tested. Cytokine and chemokine release was quantified in cell culture supernatants by commercial ELISA kits according to manufacturer's instructions. For measurements, the following kits were used: TNF (eBioscience, 88732485), IL-1ra (Abcam, ab113348), IFN- $\gamma$ (PeproTech, 900-K98), IP10 (Abcam, ab214563), and KC/Cxcl1 (R\&D Systems, DY453-05). 
Additional methods are included in Supplemental Methods.

Statistics. Mouse group sizes were determined by an a priori power analysis for a 2-tailed, 2-sample $t$ test with an $\alpha$ of 0.05 and power of 0.8 , in order to detect a $20 \%$ difference in arthritis scoring at the age of 16 weeks. Animals were randomized during the experiments. Both sex mice were used for all the experiments, and no samples or animals were excluded from analysis. The data in the graphs were analyzed for statistical significance with 2-tailed Student's $t$ test and 2-way ANOVA and Tukey's multiple comparison test, as well as with 1-way ANOVA. Bonferroni's multiple comparison tests were used to analyze groups for statistical significance. Such comparisons and the resulted $P$ values are always depicted in the figure and figure legends depending of the experiment. In each case, $P<0.05$ were considered significant. Statistical analyses in this study were performed using GraphPad Prism6 software (version 6.01). Biostatistical analysis is described in a separate section in Supplementary Material.

Study approval. Experiments were performed in the conventional unit of the animal facilities in Biomedical Sciences Research Center (BSRC) "Alexander Fleming" under specific pathogen-free conditions, in accordance with the guidance of the IACUC of BSRC "Alexander Fleming" and in conjunction with the Veterinary Service Management of the Hellenic Republic Prefecture of Attika/Greece. Experiments were monitored and reviewed throughout its duration by the respective Animal Welfare Body for compliance with the permission granted.

\section{Author contributions}

MS and GK designed research; MS, PC, LN, AP, KM, MCD, and NK performed research; MS, PC, and $\mathrm{KM}$ analyzed data; HF, VGD, and MHA supervised and conceived experiments at the GMC; and MS and GK wrote the paper.

\section{Acknowledgments}

This work was supported by the IMI project BTCure (grant 115142-2), Advanced ERC grant MCs-inTEST (grant 340217), and "Research Project for Excellence IKY/Siemens" (grant 3288) to GK, as well as an "IKY fellowship of Excellence for postgraduate studies in Greece - Siemens program" to MS and Operational Program "Competitiveness, Entrepreneurship and Innovation 2014-2020," cofinanced by Greece and the European Union (European Regional Development Fund) under action code MIS 5002562 (KRIPIS II). This work was also supported by the German Federal Ministry of Education and Research (Infrafrontier grant $01 \mathrm{KX} 1012$ to MHDA).

The authors wish to thank Vangelis Harokopos and the BSRC Al Fleming Genomics Facility for assistance with the RNA-seq experiments; Michalis Meletiou for assistance with mice genotyping and Anna Katevaini for histological sections and stainings; and Marietta Armaka and Vasiliki Koliaraki for the scientific input throughout the project. The authors also wish to thank the InfrafrontierGR Infrastructure (cofunded by the ERDF and Greek NSRF 2007-2013) for providing mouse hosting and phenotyping facilities, including transgenesis, histopathology, flow cytometry, and advanced microscopy.

Address correspondence to: George Kollias, Biomedical Sciences Research Center, "Alexander Fleming", 34 Fleming Street, 16672, Vari, Greece. Phone: 302109656310; Email: kollias@fleming.gr.

1. Michaud K, Wolfe F. Comorbidities in rheumatoid arthritis. Best Pract Res Clin Rheumatol. 2007;21(5):885-906.

2. Dougados M, et al. Prevalence of comorbidities in rheumatoid arthritis and evaluation of their monitoring: results of an international, cross-sectional study (COMORA). Ann Rheum Dis. 2014;73(1):62-68.

3. Nurmohamed MT, Heslinga M, Kitas GD. Cardiovascular comorbidity in rheumatic diseases. Nat Rev Rheumatol. 2015;11(12):693-704.

4. Wright K, Crowson CS, Gabriel SE. Cardiovascular comorbidity in rheumatic diseases: a focus on heart failure. Heart Fail Clin. 2014;10(2):339-352.

5. Voskuyl AE. The heart and cardiovascular manifestations in rheumatoid arthritis. Rheumatology (Oxford). 2006;45 Suppl 4:iv4-iv7

6. Keffer J, et al. Transgenic mice expressing human tumour necrosis factor: a predictive genetic model of arthritis. $E M B O J$. 1991;10(13):4025-4031.

7. Kontoyiannis D, Pasparakis M, Pizarro TT, Cominelli F, Kollias G. Impaired on/off regulation of TNF biosynthesis in mice lacking TNF AU-rich elements: implications for joint and gut-associated immunopathologies. Immunity. 1999;10(3):387-398.

8. Kontoyiannis D, et al. Genetic dissection of the cellular pathways and signaling mechanisms in modeled tumor necrosis factor-induced Crohn's-like inflammatory bowel disease. J Exp Med. 2002;196(12):1563-1574. 
9. Armaka M, Apostolaki M, Jacques P, Kontoyiannis DL, Elewaut D, Kollias G. Mesenchymal cell targeting by TNF as a common pathogenic principle in chronic inflammatory joint and intestinal diseases. J Exp Med. 2008;205(2):331-337.

10. Bartok B, Firestein GS. Fibroblast-like synoviocytes: key effector cells in rheumatoid arthritis. Immunol Rev. 2010;233(1):233-255

11. Bottini N, Firestein GS. Duality of fibroblast-like synoviocytes in RA: passive responders and imprinted aggressors. Nat Rev Rheumatol. 2013;9(1):24-33.

12. McInnes IB, Buckley CD, Isaacs JD. Cytokines in rheumatoid arthritis - shaping the immunological landscape. Nat Rev Rheumatol. 2016;12(1):63-68.

13. Brennan FM, Chantry D, Jackson A, Maini R, Feldmann M. Inhibitory effect of TNF alpha antibodies on synovial cell interleukin-1 production in rheumatoid arthritis. Lancet. 1989;2(8657):244-247.

14. Turiel M, et al. The heart in rheumatoid arthritis. Autoimmun Rev. 2010;9(6):414-418.

15. Avina-Zubieta JA, Thomas J, Sadatsafavi M, Lehman AJ, Lacaille D. Risk of incident cardiovascular events in patients with rheumatoid arthritis: a meta-analysis of observational studies. Ann Rheum Dis. 2012;71(9):1524-1529.

16. Momeni M, Taylor N, Tehrani M. Cardiopulmonary manifestations of ankylosing spondylitis. Int J Rheumatol. 2011;2011:728471.

17. Klingberg E, Sveälv BG, Täng MS, Bech-Hanssen O, Forsblad-d'Elia H, Bergfeldt L. Aortic Regurgitation Is Common in Ankylosing Spondylitis: Time for Routine Echocardiography Evaluation? Am J Med. 2015;128(11):1244-1250.e1.

18. Gensler LS. Axial spondyloarthritis: the heart of the matter. Clin Rheumatol. 2015;34(6):995-998.

19. Kubota T, McTiernan CF, Frye CS, Demetris AJ, Feldman AM. Cardiac-specific overexpression of tumor necrosis factor-alpha causes lethal myocarditis in transgenic mice. J Card Fail. 1997;3(2):117-124.

20. Ghosh S, et al. Left-sided cardiac valvulitis in tristetraprolin-deficient mice: the role of tumor necrosis factor alpha. Am J Pathol. 2010;176(3):1484-1493.

21. Lacey D, et al. Spontaneous retrotransposon insertion into TNF 3'UTR causes heart valve disease and chronic polyarthritis. Proc Natl Acad Sci USA. 2015;112(31):9698-9703.

22. LaHaye S, Lincoln J, Garg V. Genetics of valvular heart disease. Curr Cardiol Rep. 2014;16(6):487.

23. Mulholland DL, Gotlieb AI. Cell biology of valvular interstitial cells. Can J Cardiol. 1996;12(3):231-236.

24. Lincoln J, Garg V. Etiology of valvular heart disease-genetic and developmental origins. Circ J. 2014;78(8):1801-1807.

25. Moore-Morris T, et al. Resident fibroblast lineages mediate pressure overload-induced cardiac fibrosis. J Clin Invest. 2014;124(7):2921-2934.

26. Song K, et al. Heart repair by reprogramming non-myocytes with cardiac transcription factors. Nature. 2012;485(7400):599-604

27. Furtado MB, Nim HT, Boyd SE, Rosenthal NA. View from the heart: cardiac fibroblasts in development, scarring and regeneration. Development. 2016;143(3):387-397.

28. Brenner D, Blaser H, Mak TW. Regulation of tumour necrosis factor signalling: live or let die. Nat Rev Immunol. 2015;15(6):362-374.

29. Douni E, Kollias G. A critical role of the p75 tumor necrosis factor receptor (p75TNF-R) in organ inflammation independent of TNF, lymphotoxin alpha, or the p55TNF-R. J Exp Med. 1998;188(7):1343-1352.

30. Tsakiri N, Papadopoulos D, Denis MC, Mitsikostas DD, Kollias G. TNFR2 on non-haematopoietic cells is required for Foxp3+ Treg-cell function and disease suppression in EAE. Eur J Immunol. 2012;42(2):403-412.

31. Chatzidakis I, Fousteri G, Tsoukatou D, Kollias G, Mamalaki C. An essential role for TNF in modulating thresholds for survival, activation, and tolerance of CD8+ T cells. J Immunol. 2007;178(11):6735-6745.

32. Shiga T, Sato K, Kataoka S, Sano S. TNF inhibitors directly target Th17 cells via attenuation of autonomous TNF/TNFR2 signalling in psoriasis. J Dermatol Sci. 2015;77(1):79-81.

33. Miller PG, Bonn MB, McKarns SC. Transmembrane TNF-TNFR2 Impairs Th17 Differentiation by Promoting Il2 Expression. J Immunol. 2015;195(6):2633-2647.

34. Chen X, Oppenheim JJ. Contrasting effects of TNF and anti-TNF on the activation of effector T cells and regulatory T cells in autoimmunity. FEBS Lett. 2011;585(23):3611-3618.

35. Venkatesh D, et al. Endothelial TNF receptor 2 induces IRF1 transcription factor-dependent interferon- $\beta$ autocrine signaling to promote monocyte recruitment. Immunity. 2013;38(5):1025-1037.

36. Gao H, et al. Opposing Functions of Microglial and Macrophagic TNFR2 in the Pathogenesis of Experimental Autoimmune Encephalomyelitis. Cell Rep. 2017;18(1):198-212.

37. Prados A, Kollias G, Koliaraki V. CollagenVI-Cre mice: A new tool to target stromal cells in secondary lymphoid organs. Sci Rep. 2016;6:33027.

38. Muzumdar MD, Tasic B, Miyamichi K, Li L, Luo L. A global double-fluorescent Cre reporter mouse. Genesis. 2007;45(9):593-605

39. Furtado MB, Costa MW, Rosenthal NA. The cardiac fibroblast: Origin, identity and role in homeostasis and disease. Differentiation. 2016;92(3):93-101.

40. Honda S, et al. A novel mouse model of aortic valve stenosis induced by direct wire injury. Arterioscler Thromb Vasc Biol. 2014;34(2):270-278.

41. Zukowska P, et al. Deletion of CD73 in mice leads to aortic valve dysfunction. Biochim Biophys Acta. 2017;1863(6):1464-1472.

42. Hachicha Z, Dumesnil JG, Bogaty P, Pibarot P. Paradoxical low-flow, low-gradient severe aortic stenosis despite preserved ejection fraction is associated with higher afterload and reduced survival. Circulation. 2007;115(22):2856-2864.

43. Cubeddu LX. QT prolongation and fatal arrhythmias: a review of clinical implications and effects of drugs. Am J Ther. 2003;10(6):452-457.

44. Baumgartner $\mathrm{H}$, et al. Echocardiographic assessment of valve stenosis: EAE/ASE recommendations for clinical practice. Eur $J$ Echocardiogr. 2009;10(1):1-25.

45. Schwenk F, Baron U, Rajewsky K. A cre-transgenic mouse strain for the ubiquitous deletion of loxP-flanked gene segments including deletion in germ cells. Nucleic Acids Res. 1995;23(24):5080-5081.

46. Koliaraki V, Pasparakis M, Kollias G. IKK $\beta$ in intestinal mesenchymal cells promotes initiation of colitis-associated cancer J Exp Med. 2015;212(13):2235-2251.

47. Cushing MC, Mariner PD, Liao JT, Sims EA, Anseth KS. Fibroblast growth factor represses Smad-mediated myofibroblast activation in aortic valvular interstitial cells. FASEB J. 2008;22(6):1769-1777. 
48. Liu AC, Joag VR, Gotlieb AI. The emerging role of valve interstitial cell phenotypes in regulating heart valve pathobiology. Am J Pathol. 2007;171(5):1407-1418.

49. Latif N, et al. Modulation of human valve interstitial cell phenotype and function using a fibroblast growth factor 2 formulation. PLoS One. 2015;10(6):e0127844.

50. Wang H, Leinwand LA, Anseth KS. Cardiac valve cells and their microenvironment--insights from in vitro studies. Nat Rev Cardiol. 2014;11(12):715-727

51. Chouvardas P, Kollias G, Nikolaou C. Inferring active regulatory networks from gene expression data using a combination of prior knowledge and enrichment analysis. BMC Bioinformatics. 2016;17 Suppl 5:181.

52. Aidinis V, et al. Functional analysis of an arthritogenic synovial fibroblast. Arthritis Res Ther. 2003;5(3):R140-R157.

53. Vasilopoulos Y, Gkretsi V, Armaka M, Aidinis V, Kollias G. Actin cytoskeleton dynamics linked to synovial fibroblast activation as a novel pathogenic principle in TNF-driven arthritis. Ann Rheum Dis. 2007;66 Suppl 3:iii23-iii28.

54. Wang L, et al. Tumor necrosis factor receptor $2 /$ AKT and ERK signaling pathways contribute to the switch from fibroblasts to CAFs by progranulin in microenvironment of colorectal cancer. Oncotarget. 2017;8(16):26323-26333.

55. Zhao T, Li H, Liu Z. Tumor necrosis factor receptor 2 promotes growth of colorectal cancer via the PI3K/AKT signaling pathway. Oncol Lett. 2017;13(1):342-346.

56. Ma X, et al. Hippo signaling promotes JNK-dependent cell migration. Proc Natl Acad Sci USA. 2017;114(8):1934-1939.

57. Ma X, et al. Myc suppresses tumor invasion and cell migration by inhibiting JNK signaling. Oncogene. 2017;36(22):3159-3167.

58. Madsen PM, et al. Oligodendroglial TNFR2 Mediates Membrane TNF-Dependent Repair in Experimental Autoimmune Encephalomyelitis by Promoting Oligodendrocyte Differentiation and Remyelination. J Neurosci. 2016;36(18):5128-5143

59. Kelly ML, et al. TNF receptor 2, not TNF receptor 1, enhances mesenchymal stem cell-mediated cardiac protection following acute ischemia. Shock. 2010;33(6):602-607.

60. Probert L. TNF and its receptors in the CNS: The essential, the desirable and the deleterious effects. Neuroscience. 2015;302:2-22.

61. Kalliolias GD, Ivashkiv LB. TNF biology, pathogenic mechanisms and emerging therapeutic strategies. Nat Rev Rheumatol. 2016;12(1):49-62.

62. Li X, et al. Cardiac-specific overexpression of tumor necrosis factor-alpha causes oxidative stress and contractile dysfunction in mouse diaphragm. Circulation. 2000;102(14):1690-1696.

63. Glass AM, Coombs W, Taffet SM. Spontaneous cardiac calcinosis in BALB/cByJ mice. Comp Med. 2013;63(1):29-37.

64. De Bari C. Are mesenchymal stem cells in rheumatoid arthritis the good or bad guys? Arthritis Res Ther. 2015;17:113.

65. Sneddon JB, et al. Bone morphogenetic protein antagonist gremlin 1 is widely expressed by cancer-associated stromal cells and can promote tumor cell proliferation. Proc Natl Acad Sci USA. 2006;103(40):14842-14847.

66. Mueller KA, et al. Gremlin-1 identifies fibrosis and predicts adverse outcome in patients with heart failure undergoing endomyocardial biopsy. J Card Fail. 2013;19(10):678-684

67. Li XM, et al. Impaired TNF/TNFR2 signaling enhances Th2 and Th17 polarization and aggravates allergic airway inflammation. Am J Physiol Lung Cell Mol Physiol. 2017;313(3):L592-L601.

68. Argaw AT, Gurfein BT, Zhang Y, Zameer A, John GR. VEGF-mediated disruption of endothelial CLN-5 promotes blood-brain barrier breakdown. Proc Natl Acad Sci USA. 2009;106(6):1977-1982.

69. Victoratos P, et al. FDC-specific functions of p55TNFR and IKK2 in the development of FDC networks and of antibody responses. Immunity. 2006;24(1):65-77.

70. Furtado MB, Nim HT, Gould JA, Costa MW, Rosenthal NA, Boyd SE. Microarray profiling to analyse adult cardiac fibroblast identity. Genom Data. 2014;2:345-350.

71. Liang CC, Park AY, Guan JL. In vitro scratch assay: a convenient and inexpensive method for analysis of cell migration in vitro. Nat Protoc. 2007;2(2):329-333. 